\title{
Reproducing the Universe: a comparison between the EAGLE simulations and the nearby DustPedia galaxy sample
}

\author{
Ana Trčka ${ }^{\odot},{ }^{1 \star}$ Maarten Baes ${ }^{\odot},{ }^{1}$ Peter Camps ${ }^{\odot},{ }^{1}$ Sharon E. Meidt, ${ }^{1}$ James Trayford, ${ }^{2}$ \\ Simone Bianchi ${ }^{\oplus},{ }^{3}$ Viviana Casasola, ${ }^{3,4}$ Letizia P. Cassarà, ${ }^{5}$ Ilse De Looze ${ }^{\oplus}, 1,6$ \\ Pieter De Vis, ${ }^{7}$ Wouter Dobbels, ${ }^{2}$ Jacopo Fritz ${ }^{\odot},{ }^{8}$ Maud Galametz, ${ }^{9}$ Frédéric Galliano, ${ }^{9}$ \\ Antonios Katsianis, ${ }^{10,11}$ Suzanne C. Madden, ${ }^{9}$ Aleksandr V. Mosenkov ${ }^{\circledR},{ }^{12}$ \\ Angelos Nersesian, ${ }^{1,13,14}$ Sébastien Viaene ${ }^{1,15}$ and Emmanuel M. Xilouris ${ }^{13}$ \\ Affiliations are listed at the end of the paper
}

Accepted 2020 March 21. Received 2020 March 16; in original form 2019 October 22

\begin{abstract}
We compare the spectral energy distributions (SEDs) and inferred physical properties for simulated and observed galaxies at low redshift. We exploit UV-submillimetre mock fluxes of $\sim 7000 z=0$ galaxies from the EAGLE suite of cosmological simulations, derived using the radiative transfer code SKIRT. We compare these to $\sim 800$ observed galaxies in the UV-submillimetre range, from the DustPedia sample of nearby galaxies. To derive global properties, we apply the SED fitting code CIGALE consistently to both data sets, using the same set of $\sim 80$ million models. The results of this comparison reveal overall agreement between the simulations and observations, both in the SEDs and in the derived physical properties, with a number of discrepancies. The optical and far-infrared regimes, and the scaling relations based upon the global emission, diffuse dust, and stellar mass, show high levels of agreement. However, the mid-infrared fluxes of the EAGLE galaxies are overestimated while the far-UV domain is not attenuated enough, compared to the observations. We attribute these discrepancies to a combination of galaxy population differences between the samples and limitations in the subgrid treatment of star-forming regions in the EAGLE-SKIRT postprocessing recipe. Our findings show the importance of detailed radiative transfer calculations and consistent comparison, and provide suggestions for improved numerical models.
\end{abstract}

Key words: radiative transfer-methods: numerical-dust, extinction-galaxies: evolutiongalaxies: formation-submillimetre: galaxies.

\section{INTRODUCTION}

Despite the fact that over the last decades our knowledge of galaxy formation and evolution has improved substantially, we still have only a fragmentary understanding of all the complex and coupled physical phenomena that shape galaxies. Numerical simulations of galaxy formation and evolution (Vogelsberger et al. 2020, and references therein) are a needed and valuable tool to alleviate these difficulties, provided that they are able to reproduce galaxy populations that, in various aspects, resemble the ones found in the real Universe. Therefore, it is necessary to compare the simulated and observed objects in order to test the models and also to fine-tune the subgrid parameters.

In recent years, the power of cosmological hydrodynamical simulations increased immensely (e.g. Vogelsberger et al. 2014; Schaye et al. 2015; Pillepich et al. 2018; Davé et al. 2019). They

^E-mail: ana.trcka@ugent.be are able to reproduce galaxy properties and scaling relations that were not used for calibration, including hydrogen content, colours, morphology, and properties of satellite galaxies (Lagos et al. 2015; Sales et al. 2015; Trayford et al. 2015; Bahé et al. 2017; Crain et al. 2017; Nelson et al. 2018; Diemer et al. 2019). However, comparing simulations to observations is not trivial since the output from simulations [e.g. stellar mass, star formation rates (SFRs), metallicity, etc.] usually is not directly comparable to observational data (e.g. fluxes at various broad-bands).

Commonly, a comparison is made in the physical realm, which involves adopting different assumptions, tracers, and recipes to calculate physical properties from the observed light. This approach can introduce systematics and uncertainties, even when deriving relatively simple properties such as stellar masses and SFRs (RosaGonzález, Terlevich \& Terlevich 2002; Mitchell et al. 2013; Guidi, Scannapieco \& Walcher 2015), and even when the same method of derivation is used [e.g. spectral energy distribution (SED) fitting] but different codes (Pappalardo et al. 2016; Hunt et al. 2019). 
An alternative approach is to compare directly in the observed flux space. In contrast with the previous method, this one requires intensive treatment of the simulation data in order to obtain realistic mock observations. This method is needed if one wishes to investigate galaxy morphology (Dickinson et al. 2018; RodriguezGomez et al. 2019; Bignone et al. 2020), or extract galaxy colours (Trayford et al. 2015; Trayford et al. 2017; Nelson et al. 2018), or the whole SED (Camps et al. 2018; Liang et al. 2019; Ma et al. 2019; Katsianis et al. 2020).

To obtain the most realistic mock observations of simulated galaxies, aside from stars and gas, it is necessary to also include interstellar dust in the modelling. During the last decades, we have grown to understand the importance of cosmic dust as a powerful medium for distorting stellar light in galaxies (Calzetti, Kinney \& Storchi-Bergmann 1994; Galliano, Galametz \& Jones 2018). Dust reprocesses more than 30 per cent of stellar radiation of typical starforming galaxies, entirely reshaping galaxy SEDs through processes of absorption, scattering and then re-emission at longer wavelengths (Popescu \& Tuffs 2002; Skibba et al. 2011; Viaene et al. 2016; Bianchi et al. 2018). Thus, despite its low-mass fraction of less than 1 per cent of the interstellar medium (ISM) mass (Rémy-Ruyer et al. 2014), dust is a crucial ingredient in the Universe. Nevertheless, dust is still rarely modelled in cosmological simulations.

One approach adopted is to incorporate dust creation, growth, destruction, and dynamics into simulations directly (McKinnon, Torrey \& Vogelsberger 2016; McKinnon et al. 2017; Aoyama et al. 2018, 2019; Davé et al. 2019; Hou et al. 2019). This, however is a very computationally expensive method, involving many processes that remain poorly understood. A simpler method, that can be applied more easily to large-scale cosmological simulations, is to model dust based on information on gas and stars from the simulation (Camps et al. 2016; Trayford et al. 2017; Liang et al. 2018; Narayanan et al. 2018a; Cochrane et al. 2019; Ma et al. 2019; Rodriguez-Gomez et al. 2019), and then perform radiative transfer in post-processing

EAGLE (Schaye et al. 2015; Crain et al. 2015) is a suite of stateof-the-art cosmological hydrodynamical simulations. Since these simulations do not include dust in the modelling, assumptions are needed to run the radiative transfer simulations. Camps et al. (2016) and Trayford et al. (2017) introduced a radiative transfer postprocessing procedure of the EAGLE simulations using the SKIRT code (Baes et al. 2011), and they tested how well the coupling of EAGLE and SKIRT recreates infrared (IR) and submillimetre as well as ultraviolet (UV) and optical observations of the Local Universe, respectively. They calibrated the parameters associated with the post-processing procedure using three different scaling relations to achieve the best agreement between a sub-sample of around 300 galaxies from the Herschel Reference Survey (HRS; Boselli et al. 2010; Cortese et al. 2012) and a $K$-band luminosity matched sample of EAGLE galaxies. Since these studies showed broad agreement with the observations, Camps et al. (2018) enriched the public EAGLE data base (McAlpine et al. 2016) with the mock fluxes for most of the EAGLE galaxies, based on the same modelling prescriptions as before.

There are, however, a couple of caveats. The HRS sample is limited in size, and centred on the Virgo Cluster and thus may be less representative for the general galaxy population. Also, dust and stellar masses, which Camps et al. (2016) used for the calibration, were derived adopting simple recipes using only SPIRE data and the SDSS $g$ and $i$ bands, respectively, so in total only five bands. Therefore, the calibration did not take into account data at far-UV (FUV) and mid-infrared (MIR) wavelengths, which are crucial for the calculation of physical parameters such as stellar mass and SFR and which depend critically on the properties and distribution of the dust (Bell et al. 2003; Kennicutt \& Evans 2012).

In their recent work, Baes et al. (2019) argue that the described EAGLE-SKIRT coupling broadly reproduces the Galaxy And Mass Assembly (GAMA; Driver et al. 2011; Liske et al. 2015) cosmic $\mathrm{SED}^{1}$ at $z=0$ (Andrews et al. 2017). However, the comparison shows tension at UV wavelengths, revealing that the attenuation by the EAGLE-SKIRT model at these wavelengths is underestimated. This indicates discrepancy in the galaxy populations between samples, or the need to improve the radiative transfer postprocessing recipe. The analysis of the cosmic SEDs alone, however, is insufficient to fully understand the cause and the treatment of the potential issue. To achieve this one has to analyse individual galaxies.

The aim of this paper is to compare the EAGLE simulated galaxies to observed galaxies in the nearby Universe, and in particular to verify the calibration of the EAGLE-SKIRT procedure, developed by Camps et al. (2016) and Trayford et al. (2016), and extended by Camps et al. (2018). We perform the analysis at low redshifts, since nearby galaxies benefit from higher signal-to-noise data, enabling detailed characterization across wavelength. As a comparison sample, we use DustPedia, the largest sample of nearby galaxies with matched aperture photometry in more than 40 bands from UV to millimetre wavelengths (Clark et al. 2018). The main advantages over the HRS sub-sample, which was used in the original EAGLE-SKIRT calibration, are the larger spread in environment and almost three times higher number of galaxies (Davies et al. 2017). In this paper, we exploit advantages of both comparative approaches, by comparing the samples in the two domains: of the observed fluxes and of physical properties. We use the fluxes from the post-processing of the EAGLE galaxies, which together with the observed DustPedia fluxes we treat in the same SED fitting environment of the CIGALE code (Boquien et al. 2019). Taking into account information across the entire UV-submillimetre wavelength range, and with the same assumptions and model parameters for both simulations and observations, we derive physical properties, and therefore compare these samples in a consistent way.

We organize the paper as follows. In Section 2, we briefly review the EAGLE simulations, our SKIRT post-processing procedure, the DustPedia sample, and the CIGALE SED fitting procedure. In Section 3, we perform the comparison between EAGLE-SKIRT simulations and the DustPedia sample of nearby galaxies. In Section 3.1, we analyse relations between the observational and mock fluxes, prior to SED fitting. This provides some insight into differences between the samples which we analyse in more depth when comparing the SEDs in Section 3.2. Physical properties derived from our CIGALE fitting are presented in Section 3.3. Our results are discussed in Section 4 and summarized in Section 5.

\section{METHODS}

\subsection{The EAGLE simulation suite}

We summarize the characteristics of the EAGLE simulations relevant to our study, but refer to Schaye et al. (2015) and Crain et al. (2015) for full details. The EAGLE suite consists of cosmological hydrodynamical simulations with different resolutions, subgrid

\footnotetext{
${ }^{1}$ Total energy in a cosmologically representative volume at different wave-
} lengths. 
Table 1. List of relevant properties: name; box size of the simulation in comoving Mpc $(L)$; number of particles $(N)$; gas particle initial mass $\left(m_{\mathrm{g}}\right)$; and maximum proper gravitational softening length $\left(\epsilon_{\text {prop }}\right)$.

\begin{tabular}{lcccc}
\hline Simulation name (label) & $\begin{array}{c}L \\
(\mathrm{cMpc})\end{array}$ & $N$ & $\begin{array}{c}m_{g} \\
\left(\mathrm{M}_{\odot}\right)\end{array}$ & $\begin{array}{c}\epsilon_{\text {prop }} \\
(\mathrm{kpc})\end{array}$ \\
\hline Ref-L1001504 (Ref100) & 100 & $1504^{3}$ & $1.81 \times 10^{6}$ & 0.70 \\
Recal-L025N0752 (Recal25) & 25 & $752^{3}$ & $2.26 \times 10^{5}$ & 0.35 \\
\hline
\end{tabular}

models and a range of box sizes up to 100 comoving $^{2} \mathrm{Mpc}$ on a side. The simulations were performed using a modified version of the $\mathrm{N}$-body Tree-PM smoothed particle hydrodynamics code GADGET 3 (Springel 2005). The adopted cosmology is lambda cold dark matter with parameters constrained by the Planck mission (Planck Collaboration XVI 2014). The assumed stellar initial mass function (IMF) is that of Chabrier (2003).

All simulations incorporate subgrid models for radiative cooling, star formation, stellar evolution and enrichment, black hole seeding and growth, stellar and active galactic nuclei (AGNs) feedback. Free subgrid parameters were calibrated to reproduce the observed $z=0.1$ galaxy stellar mass function, galaxy size, and the relation between the black hole and stellar mass of galaxies.

For this study, we are focusing on the redshift $z=0$ and on two EAGLE simulations: The reference Ref-L100N1504 (hereafter called the Ref-100 simulation) and Recal-L025N0752 (hereafter Recal-25). Recal-25 has higher resolution and, appropriately, a different set of subgrid recipes than Ref-100. Hence, regarding 'weak' convergence (recalibration of subgrid physics as a consequence of a changed resolution, see section 2.2 in Schaye et al. 2015), these simulations are comparable. The main properties of Ref-100 and Recal-25 are listed in Table 1. The last two columns represent mass and spatial resolutions.

\subsection{Post-processing EAGLE with SKIRT}

SKIRT is a state-of-the-art Monte Carlo radiative transfer code (Baes et al. 2003, 2011; Camps \& Baes 2015) that incorporates all relevant processes between dust and radiation in a galaxy (absorption, scattering, dust emission, and dust self-absorption). One of its features is the capability to calculate mock observations from the snapshot data of a hydrodynamical simulation. The method is described in Camps et al. (2016) and Trayford et al. (2017). In order to expand these studies to higher redshifts, Camps et al. (2018), employed a slightly modified method, calculating mock fluxes for all galaxies above a stellar mass threshold of $10^{8.5} \mathrm{M}_{\odot}$ for 6 EAGLE simulations described in Schaye et al. (2015). Here, we list the most relevant aspects of the method and the galaxy sample.

Camps et al. (2016) and Trayford et al. (2017) chose the value for the stellar mass threshold of $10^{8.5} \mathrm{M}_{\odot}$ to have at least 100 star particles (below this value, sampling effects become dominant). They extracted gas and star particles enclosed within $30 \mathrm{kpc}$ (to approximate a Petrosian aperture), as suggested in Schaye et al. (2015) and Crain et al. (2015). Galaxies at redshift $z=0$ were placed at $20 \mathrm{Mpc}$. In this study, for the intermediate resolution run Ref-100 we impose a higher stellar mass threshold of $10^{9} \mathrm{M}_{\odot}$, to ensure our sample contains sufficiently resolved galaxies. With the additional dust cut explained below, the minimum number of stellar particles for Ref-100 (Recal-25) is 1100 (2576).

\footnotetext{
${ }^{2}$ The length does not change due to space expansion.
}

For each stellar particle, the SED was acquired from the GALEXEV library (Bruzual \& Charlot 2003), based on age, metallicity and initial mass of the particle.

Since dust is not modelled in the EAGLE simulations, postprocessing of the input data (stellar and gas particles) was required. The model includes two dust sources: star-forming regions (not resolved in the EAGLE simulations), and diffuse dust. The AGN effects were not modelled.

To acquire 'star-forming particles' from the EAGLE data, Camps et al. (2016) and Trayford et al. (2017) first select star particles younger than $100 \mathrm{Myr}$ and star-forming gas particles. They resample each star-forming particle and assign formation times based on the SFR of the parent particle: those with formation times lower than 10 Myr stay in the star formation particle bin, those with higher ones are moved to a star particle bin, whereas those not formed are moved to a gas particle bin. Then an SED from the MAPPINGS-III family (Groves et al. 2008) was assigned to each starforming particle, based on its SFR, metallicity, pressure of the ISM, compactness and $f_{\mathrm{PDR}}$, representing the covering fraction of the photodissociation regions (PDRs).

The diffuse dust distribution was derived from the distribution of gas. The assumed dust model was from Zubko, Dwek \& Arendt (2004), which consists of bare graphite and silicate grains, and polycyclic aromatic hydrocarbon (PAH) molecules, and uses solar ISM abundances. The dust mass was derived from cool or starforming gas, and depends on the fraction of metals in dust $f_{\text {dust }} \cdot{ }^{3}$

The post-processing pipeline had two free parameters: $f_{\text {dust }}$ and $f_{\text {PDR }}$. Camps et al. (2016) selected the values for these parameters based on three scaling relations: the submillimetre colour diagram, specific dust mass $\left(M_{\text {dust }} / M_{\text {star }}\right)$ versus stellar mass, and $M_{\text {dust }} / M_{\text {star }}$ versus the NUV $-r$ colour. The comparison was performed between galaxies from the HRS sub-sample and a matched sample of about 300 EAGLE galaxies. The adopted value of the covering fraction is $f_{\mathrm{PDR}}=0.1$ (below the reference value of Jonsson; Groves \& Cox 2010). They also adopt a metal fraction $f_{\text {dust }}$ of 0.3 (Dwek 1998; Brinchmann et al. 2013). Following, Camps et al. (2018) slightly changed the procedure. First, they incorporated the process of dust self-absorption. Secondly, since the number of EAGLE galaxies is rather large, the radiative transfer procedure only included the calculation of the broad-band fluxes, and Camps et al. (2018) did not generate resolved images for each individual galaxy. Considering the images are not produced, the effects of the observational limits (e.g. surface brightness sensitivity limits of the telescope), are not accounted for. Camps et al. (2018) applied the procedure on three different angles: face-on, edge-on, and random. In this study, we use only the random angle which corresponds to the original galaxy orientation in the simulation. This way we mimic the random orientation in the observed galaxy sample.

The EAGLE mock data used in this paper (dust-attenuated and dust emission fluxes) are from Camps et al. (2018) and they are extracted from the public data base ${ }^{4}$ (McAlpine et al. 2016).

It was already indicated in Camps et al. (2016), and then confirmed in Camps et al. (2018) that the post-processing procedure produces unphysically low dust temperatures, for a fraction of simulated galaxies (see fig. 3 in Camps et al. 2018). The cause of this is that these galaxies have insufficiently resolved dust distribution to characterize a realistic dust-to-stellar geometry. Therefore, from our

\footnotetext{
${ }^{3}$ Defined as $\frac{\rho_{\text {dust }}}{Z \rho_{\text {gas }}}$, with $Z, \rho_{\text {dust }}$, and $\rho_{\text {gas }}$ as metallicity, dust, and gas density, respectively.

${ }^{4}$ http://icc.dur.ac.uk/Eagle/database.php
} 

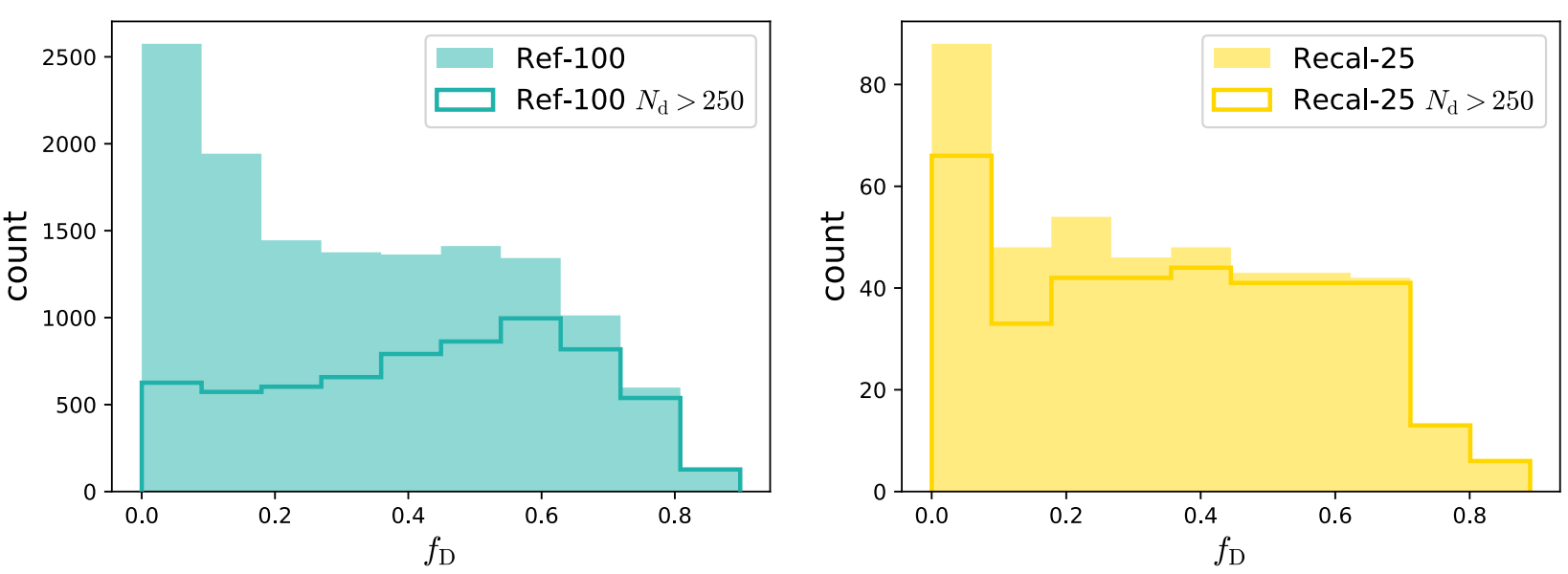

Figure 1. Distribution of the disc stellar mass fraction for EAGLE at $z=0$. Left-hand panel shows results for Ref- $100\left(M_{\text {star }}>10^{9} \mathrm{M}_{\odot}\right)$ and right for Recal-25 $\left(M_{\text {star }}>10^{8.5} \mathrm{M}_{\odot}\right)$. Filled histograms indicate the original EAGLE sample while outlined indicate our sample with resolved dust, i.e. with the number of dust particles above 250 .

samples we exclude galaxies that have less than 250 dust particles, as suggested by Camps et al. (2018). As a consequence our Ref-100 sample is 50 per cent smaller and Recal-25 is 15 per cent smaller than the original EAGLE samples. To understand which galaxy type is mostly affected by this dust cut, we exploited the morphology data of the EAGLE galaxies (Trayford et al. 2019). We analysed the disc stellar mass fraction $f_{\mathrm{D}}$, a parameter defined as $1-$ bulge-to-total mass ratio, with the bulge defined as twice the mass of the counterrotating stellar particles (Abadi et al. 2003). In Fig. 1, we compared distributions of $f_{\mathrm{D}}$ of our sample and the whole EAGLE sample $\left[\log M_{\mathrm{star}} / \mathrm{M}_{\odot}>8.5\right.$ (9) for Recal-25 (Ref-100), and at $z=0$ for both simulation runs]. Not surprisingly, our Ref-100 sample lacks most of the elliptical galaxies (those with $f_{\mathrm{D}} \lesssim 0.5$ ), which are mainly red galaxies with low SFR (see fig. 4 in Camps et al. 2018).

\subsection{DustPedia}

DustPedia (Davies et al. 2017) is a European project initiated in order to improve our knowledge of cosmic dust and its role in the Local Universe. The DustPedia sample contains 875 nearby galaxies, observed with Herschel's PACS or SPIRE instruments (Griffin et al. 2010; Pilbratt et al. 2010; Poglitsch et al. 2010). For a nearby, but still diverse, sample of galaxies populating different environments, objects are selected to have radial velocities below $3000 \mathrm{~km} \mathrm{~s}^{-1}$. Additionally, all galaxies have at least $5 \sigma$ WISE $3.4 \mu \mathrm{m}$ flux detection. In addition to the Herschel data, the DustPedia data base ${ }^{5}$ also includes data from GALEX (Morrissey et al. 2007), SDSS (York et al. 2000), 2MASS (Skrutskie et al. 2006), WISE (Wright et al. 2010), Spitzer (Werner et al. 2004), Planck (Planck Collaboration I 2011), and IRAS (Neugebauer et al. 1984).

Clark et al. (2018) presented an aperture-matched photometry of the whole DustPedia galaxy sample, except for Planck and IRAS because of their poor resolution. The photometry for the EAGLE galaxies is derived in a similar manner with the same aperture for all bands. The average aperture for DustPedia is $17.6 \mathrm{kpc}$, which is below the value adopted for the EAGLE galaxies of $30 \mathrm{kpc}$. However, this difference is not affecting our analysis because twice the average stellar half-mass radius for the Ref-100 (Recal-25)

${ }^{5} \mathrm{http} / / /$ dustpedia.astro.noa.gr/
Table 2. Main characteristics of the two EAGLE galaxy samples and the DustPedia sample: the total number of galaxies, the aperture size, the distance from the galaxies, and the number of available bands for all the samples. For the EAGLE samples, the values are the same for every galaxy, while we provide the 16-84 per cent range and mean values for the DustPedia galaxies.

\begin{tabular}{lcccc}
\hline Galaxy sample & $N_{\text {gal }}$ & $\begin{array}{c}\text { Aperture } \\
(\mathrm{kpc})\end{array}$ & $\begin{array}{c}\text { Distance } \\
(\mathrm{Mpc})\end{array}$ & $N_{\text {bands }}{ }^{a}$ \\
\hline Ref-100 & 6593 & 30 & 20 & 29 \\
Recal-25 & 369 & 30 & 20 & 29 \\
DustPedia & 814 & {$[7.7,26.3]$} & {$[12,33]$} & {$[16,24]$} \\
& & $\langle 17.6\rangle$ & $\langle 21.5\rangle$ & $\langle 20\rangle$ \\
\hline
\end{tabular}

${ }^{a}$ Only bands with a positive flux are included.

simulation is $11 \mathrm{kpc}(8.5 \mathrm{kpc})$. This means most of the galaxies in our sample would be captured by a $17.6 \mathrm{kpc}$ aperture. Additionally, the small DustPedia apertures correspond to low stellar mass galaxies. If we inspect only the galaxies with $M_{\text {star }}>10^{8.5} \mathrm{M}_{\odot}$ (as those are more comparable with our EAGLE samples), we have a mean aperture of $18.8 \mathrm{kpc}$.

We do not include galaxies that have contamination from a nearby source, imagery artefacts or lack essential bands to constrain the SED fitting (e.g. bands in the optical and FIR). Our final DustPedia sample contains 814 galaxies. Basic information about the three galaxy samples are shown in Table 2 .

\subsection{CIGALE}

We rely on the CIGALE fitting code (version 0.12.1) (Noll et al. 2009; Boquien et al. 2019) to perform the SED fitting and derive physical properties such as the stellar and dust mass, SFR, dust luminosity, etc. CIGALE incorporates stellar, nebular, AGNs, and dust emission and dust attenuation. It contains an implementation of a delayed and truncated star-formation history (SFH) (Ciesla et al. 2016), Bruzual \& Charlot (2003) simple stellar population (SSP) libraries using Salpeter (1955) IMF, modified Calzetti et al. (2000) attenuation law, and the THEMIS (Jones et al. 2017) dust model. The resulting library has of over 80 million model SEDs. For details on the selection of modules, fitting process, parameter space and results for the DustPedia galaxies, we refer to Nersesian et al. (2019) and their table 1. 
The presence of an AGN or a strong jet can affect the SED of a host galaxy, with the highest contribution in the IR part of the spectrum (Mullaney et al. 2011; Xilouris et al. 2004, Viaene et al., in preparation). The DustPedia sample contains 19 galaxies with a high probability of hosting an AGN (Bianchi et al. 2018) and four jet-dominated galaxies (Nersesian et al. 2019). Since these galaxies account for only a small percentage of the sample, the additional CIGALE modules were not included to avoid computational costs (Nersesian et al. 2019). Since the AGNs are not modelled in the post-processing of EAGLE, we use also only non-AGN templates for the EAGLE galaxies. However, these DustPedia sources will be indicated separately on our plots.

The EAGLE data base already contains stellar mass and SFR information but we choose to re-derive these properties using CIGALE to compare the simulated and real samples in a consistent approach.

We note that a different IMF was used for the creation of the EAGLE simulations (as described in Section 2.1), and for the SED fitting. The differences in the IMFs do not affect the comparisons we perform here, except the absolute values of the stellar mass and SFR, which are $\sim 0.2$ dex higher when derived with the use of the Salpeter (1955), than with the Chabrier (2003) IMF.

An additional caveat is that the dust model adopted in the postprocessing (Zubko et al. 2004, see Section 2.2), differs from the one used in the SED fitting (THEMIS). The main differences between the two models are (1) the FIR-submillimetre emissivity is about a factor of 2 higher for the THEMIS model; (2) a couple of aromatic bands around $20 \mu \mathrm{m}$ are accounted for only in the model by Zubko et al. (2004); (3) the strength of the aromatic features relative to the FIR pick emission is two times higher in the THEMIS model compared to the model by Zubko et al. (2004), see fig. 4b of Galliano et al. (2018). We discuss in later sections how these differences affect our comparison. We chose not to change the IMF, the dust model or any other parameter in the SED modelling, for the consistency, and easier comparison with the previous studies of the DustPedia galaxies (Bianchi et al. 2018; Clark et al. 2018; De Vis et al. 2019; Nersesian et al. 2019; Dobbels et al. 2020; Casasola et al. 2020, etc.). Additionally, the same CIGALE fitting procedure for the EAGLE galaxies is already published in Baes et al. (2019). For the same reason we also use the same set of bands (i.e. all but Planck bands, since they are not included in the public EAGLE data base. We do not expect their absence would affect the SED fitting since every EAGLE galaxy has the FIR/submm region covered with all SPIRE bands.).

We present an example of a galaxy taken from each of the three samples (Ref-100, Recal-25 and DustPedia) with their respective images, ${ }^{6}$ fluxes and fitted SEDs in Fig. 2. For all three galaxies, the model SED provides a good match to the data. This is captured in Fig. 3, where we show the median deviation between the observed/mock fluxes and the fitted CIGALE fluxes for each of the three galaxy samples. The highest deviation for the samples occurs in the IRAS $60 \mu \mathrm{m}$ band (0.05-0.1 dex) and, only for the EAGLE galaxies, in the WISE $12 \mu \mathrm{m}$ band $(\sim 0.1 \mathrm{dex})$. However, most of the model bands deviate by less than 0.05 dex from the data. As an additional test of the robustness of the fit, we performed the mock analysis described in Appendix A. As follows from these tests, most of the physical properties are well constrained.

${ }^{6}$ These are obtained from the public EAGLE data base and the Sloan Digital Sky Survey.

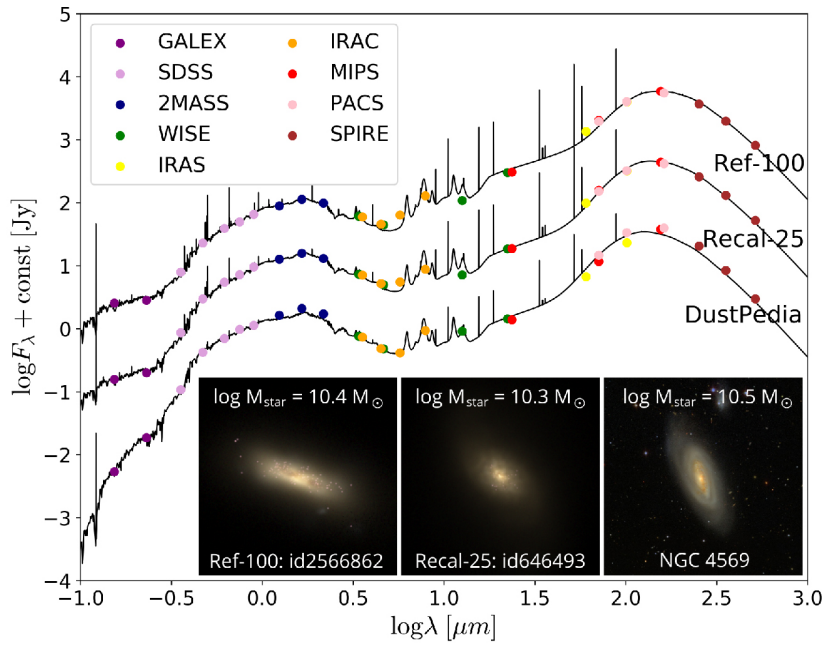

Figure 2. Example of SED data and fits derived by CIGALE of an arbitrarily selected galaxy for each galaxy sample. Images in gri bands and the stellar masses of the same galaxies are also shown.

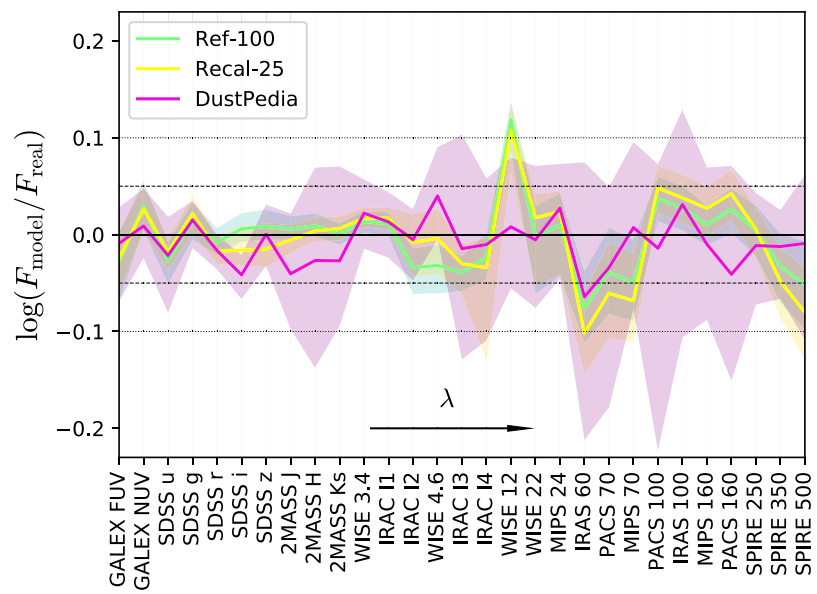

Figure 3. Ratio of the best model fluxes and the real fluxes, for the whole sample of observed and mock galaxies. Solid lines represent the median value for each band and the shaded regions show the 16-84 percent range.

To validate the CIGALE method, we plot the relation between values of different properties obtained using CIGALE and the intrinsic EAGLE simulation values. Results are presented in Fig. 4. A constant offset is present, however, most of the data do not deviate more than 0.2 dex. The main cause of the difference are the assumptions we made about the star formation histories and the use of different IMFs.

All physical properties presented in this paper, unless otherwise stated, are derived from the CIGALE fits.

\section{RESULTS}

\subsection{MIR and FIR luminosities and colours}

We start first with an inspection of the fluxes from the DustPedia and the EAGLE data bases, prior to the SED fitting. The four scatter plots of Fig. 5 already highlight the reassuring agreement between the real and mock fluxes in the NIR to submillimetre regime. The relations for the EAGLE galaxies appear to have less scatter, than those for the DustPedia galaxies, which is expected since the EAGLE 

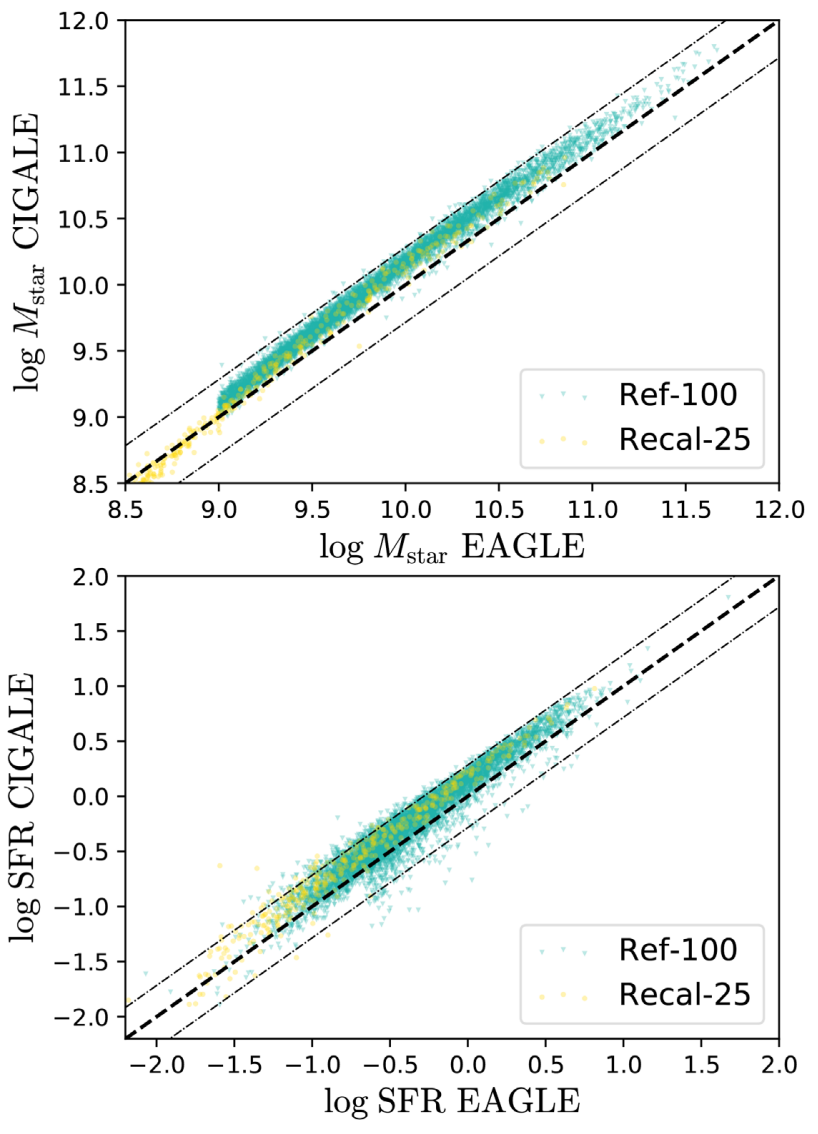

Figure 4. Comparison between the property values derived using CIGALE and the intrinsic simulation values. Dashed line represents one-to-one relation while the dash-dotted lines indicates the \pm 0.2 dex deviation.

galaxies are modelled to all have the same dust properties and no observational limitations (see Section 2.2).

The top left panel of Fig. 5 shows the relation between the WISE $22 \mu \mathrm{m}$ and WISE $3.4 \mu \mathrm{m}$ luminosity. As the WISE $3.4 \mu \mathrm{m}$ band is a good proxy for the stellar mass (Wen et al. 2013) and WISE $22 \mu \mathrm{m}$ band for the SFR (Lee, Hwang \& Ko 2013), this relation is a proxy of the main sequence of star-forming galaxies (Noeske et al. 2007). The main sequence of the EAGLE galaxies has already been widely investigated. Schaye et al. (2015), Furlong et al. (2015), and Katsianis, Tescari \& Wyithe (2016) compared the simulations with observations by Bauer et al. (2013) within the GAMA survey. They all find that while the simulations typically produce specific SFRs (sSFRs) around 0.2 dex below the observational relation for the star-forming galaxies, the agreement is within the errors, with the best agreement for $M_{\text {star }}>10^{10} \mathrm{M}_{\odot}$ and for the high-resolution simulations. In our study, in general, all three samples follow the same trend, but with the EAGLE galaxies above the DustPedia relation. The dearth of EAGLE galaxies at lower luminosities is a consequence of the stellar mass threshold, as discussed in Section 2.2. A bimodality, representing blue and red galaxies, is reproduced: the cloud with the higher $L_{22}$ for the same $L_{3.4}$ (higher SFR for the same stellar mass) represents blue galaxies and the lower $L_{22}$ cloud red galaxies. We label a galaxy as blue or red based on its position on a $u-r$ versus $r-z$ colour-colour diagram, with the cut-off values from Chang et al. (2015). The EAGLE Ref-100 (Recal-25) sample contains 92 per cent (95 per cent) blue galaxies while DustPedia contains 50 per cent, which explains why the bimodality is not so prominent for the EAGLE samples. Also, the running medians show an offset $(\sim 0.3$ dex $)$ between EAGLE and DustPedia suggesting that EAGLE galaxies have either high $22 \mu \mathrm{m}$ or low stellar mass $\left(L_{3.4}\right)$ relative to the observed sample.

The top right panel represents the relation between dust and stellar mass proxies (SPIRE $250 \mu \mathrm{m}$ and WISE $3.4 \mu \mathrm{m}$ luminosities, respectively). A strong correspondence between both the EAGLE and the DustPedia relations is found, suggesting that the offset in the top left plot is primarily driven by the $L_{22}$ output by the EAGLESKIRT post-processing being too high. Additionally, the bimodal distribution of the DustPedia galaxies is well reproduced by the simulations, revealing that the blue, dusty galaxies form a sequence and the red, dust-poor galaxies are located in the cloud below it.

The bottom left panel shows the relationship between SPIRE $250 \mu \mathrm{m}$ and WISE $22 \mu \mathrm{m}$ luminosities. Due to our selection bias, EAGLE galaxies with little dust and low SFR are absent. The slope of the median for DustPedia is flatter for the higher $L_{22}$, which slightly changes if galaxies with AGNs are removed. Although the relation is fairly tight, again EAGLE galaxies show a discrepancy, having higher $L_{22}$ for the same $L_{250}$. This is also clearly visible in the bottom right panel of Fig. 5, where we show the relation between tracers of the specific dust mass and sSFR. The break in the median trend at $\log L_{22} / L_{3.4} \approx-0.7$ for the EAGLE samples illustrates the lack of the EAGLE galaxies with low SFR and/or high stellar mass.

In summary, at the limited number of wavelengths we study in this section, the relations between EAGLE luminosities derived from SKIRT broadly reproduce observations. The discrepancies are mostly coming from high $L_{22}$ for both EAGLE samples. To understand the origin of this deviation we continue our analysis in more depth in next sections.

\subsection{Spectral energy distributions}

Complete FUV to submillimetre SEDs for all three samples (Ref100, Recal-25, DustPedia) are extracted from the CIGALE bestmodel fits. First, we compare the SEDs, normalized by their bolometric luminosity and averaged, to gain insight into the sample properties.

Fig. 6 shows the median SEDs $^{7}$ and the regions between the 16th and 84th percentiles. Both the stellar unattenuated (left) and the stellar attenuated (right) plots are derived from the CIGALE best fits. The left-hand panel represents the intrinsic stellar radiation (if there were no dust). The shape of the median SEDs between the samples is similar, although the DustPedia sample shows a wider variety of SEDs compared to the EAGLE sample, especially at UV wavelengths. Also, the galaxies from the EAGLE samples are intrinsically slightly bluer, i.e. they emit slightly more UV and slightly less near-IR (NIR) radiation compared to the DustPedia galaxies.

The right-hand panel shows radiation re-processed and re-emitted by dust. Compared to the intrinsic SEDs, the differences between the attenuated SEDs are much more prominent. The shape is similar in the optical and submillimetre region, but the overall spread indicates that the DustPedia sample is more diverse than both the EAGLE samples. This discrepancy is expected to some extent, since all EAGLE galaxies are modelled to have the same optical and calorimetric dust properties and the same dust-to-metal ratio. Additionally, the EAGLE galaxies have much less UV attenuation

\footnotetext{
${ }^{7}$ Ten percent of the most irregular SEDs are excluded from all samples, following Bianchi et al. (2018) and Nersesian et al. (2019).
} 

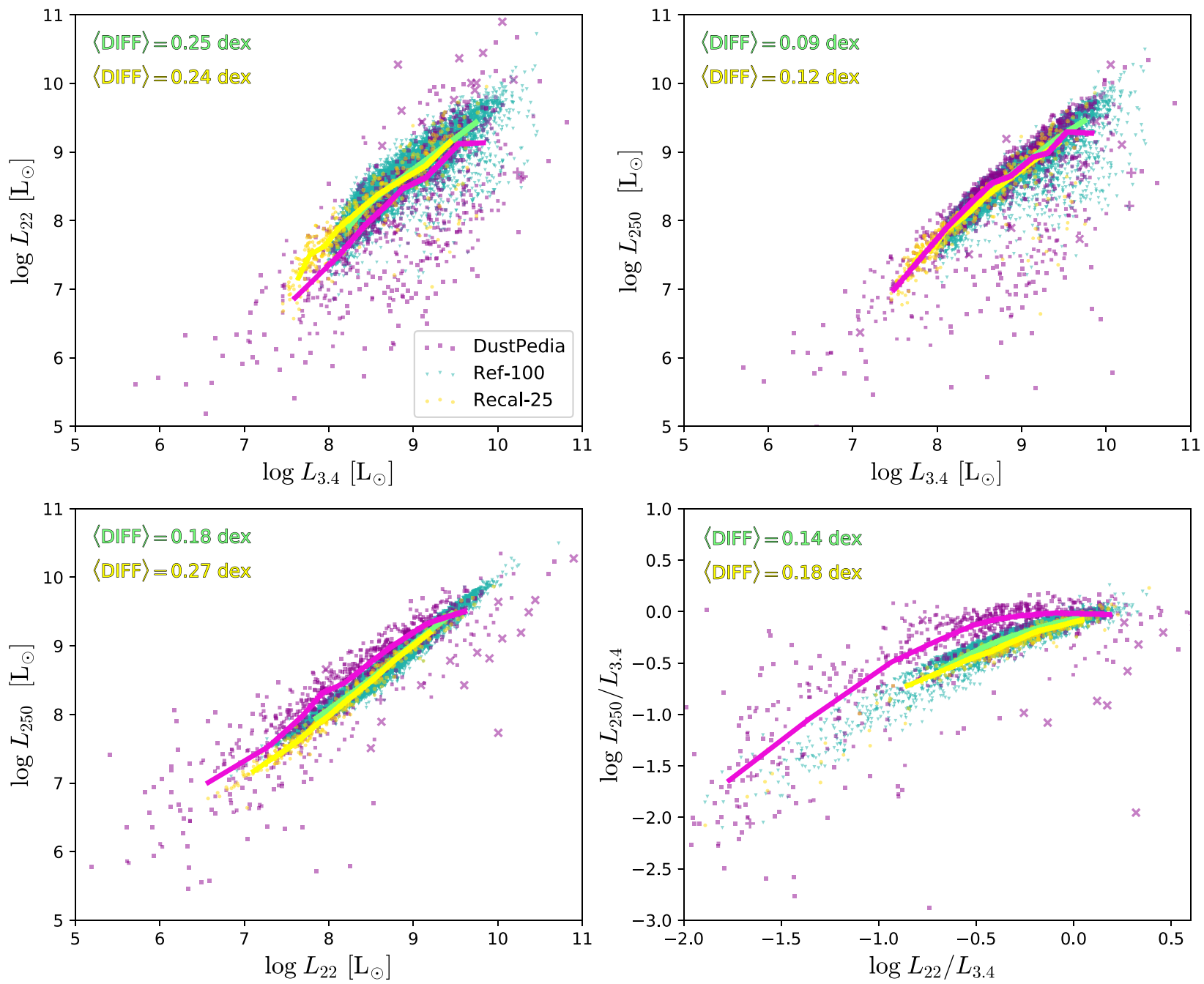

Figure 5. Relations between: (top left) WISE $22 \mu \mathrm{m}$ and WISE $3.4 \mu \mathrm{m}$ luminosities; (top right) SPIRE $250 \mu \mathrm{m}$ and WISE $3.4 \mu \mathrm{m}$ luminosities; (bottom left) SPIRE $250 \mu \mathrm{m}$ and WISE $22 \mu \mathrm{m}$ luminosities; (bottom right). The ratios of SPIRE $250 \mu \mathrm{m}$ and WISE $22 \mu \mathrm{m}$ with WISE $3.4 \mu \mathrm{m}$ luminosity. The coloured lines represent running median. The numbers in green (yellow) indicate the average offset between the running medians for Ref-100 and DustPedia (Recal-25 and DustPedia). Galaxies with an AGN (strong jet) are marked with a ' $\times$ ' ('+').

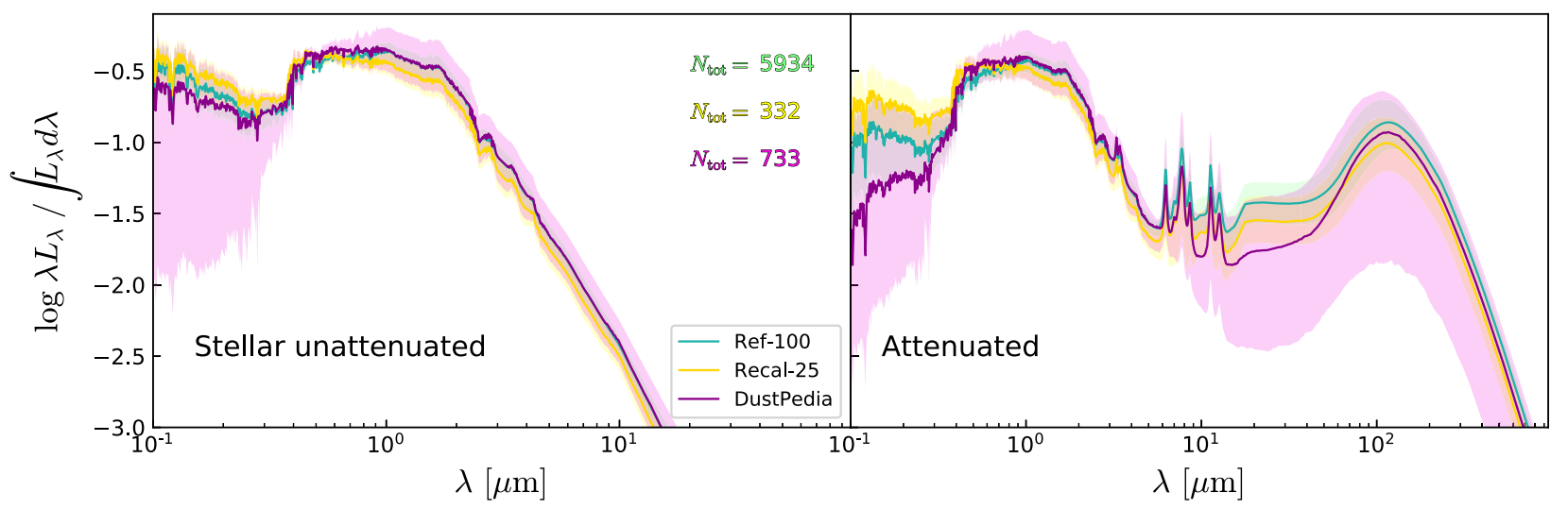

Figure 6. Median unattenuated (left-hand panel) and attenuated (right-hand panel) SEDs of each data set. The plots are reconstructed from the CIGALE best fits. Ten per cent of the galaxies with the most irregular SEDs are removed from each sample. $N_{\text {tot }}$ represents the actual number of galaxies used to derive these figures. The shaded regions represent range $16-84$ per cent. 
and more MIR radiation (see also Baes et al. 2019, their fig. 1). In the FUV band the difference between DustPedia and Ref-100 (Recal-25) is $0.37 \mathrm{dex}(0.59 \mathrm{dex})$. The difference in the WISE $22 \mu \mathrm{m}$ band is slightly lower: $0.34 \mathrm{dex}(0.21 \mathrm{dex})$. We shall return to these differences in the SEDs in Section 4.1.

\subsection{Physical properties and dust scaling relations}

In this section, we examine how well the physical properties, derived using CIGALE, are represented by their common proxies, and then we focus on the scaling relations between different physical properties.

Fig. 7a demonstrates the quality of the WISE $3.4 \mu \mathrm{m}$ luminosity as a stellar mass tracer, since it is sensitive to the evolved stellar populations that dominate the baryonic mass in galaxies, and at the same time it is not very affected by the dust attenuation (Norris et al. 2014). Solid lines represent linear fits to the data while the dashed line is the fit from Wen et al. (2013). The three samples agree very well, part from the low stellar mass end which is below the EAGLE mass thresholds. The Spearman rank-order correlation analysis coefficient (indicated in the plots) is slightly higher for the EAGLE sample than for the DustPedia sample.

Considering that most of the dust is in the cold phase, submillimetre radiation can be used as a proxy for the dust mass in galaxies (Dunne et al. 2011; Eales et al. 2012). Fig. 7b shows the level of agreement of our samples. The highest Spearman coefficient is for Recal-25. The linear fits agree with those of Dunne et al. (2011). The lack of simulated galaxies with low SPIRE $250 \mu \mathrm{m}$ luminosities is, again, caused by our chosen selection of EAGLE galaxies (see Section 2.2).

Fig. 7c shows the relation between the WISE $22 \mu \mathrm{m}$ luminosity and the SFR. From the abundance of different SFR tracers (e.g. Hao et al. 2011; Cortese et al. 2012; Lee et al. 2013; Boquien et al. 2016; Casasola et al. 2017), in this study, we are focusing on a reliable single MIR band SFR tracer (Calzetti et al. 2010; Cluver et al. 2017). Fig. 7c highlights the differences between the EAGLE and the DustPedia samples. The lower Spearman coefficient of the DustPedia sample is, together with the slope and the scatter of the relation, primarily driven by the large number of galaxies with low SFRs and luminosities at $22 \mu \mathrm{m}$. In the domain below $10^{8} \mathrm{~L}_{\odot}$, the spread in SFR is almost two orders of magnitude, at fixed $22 \mu \mathrm{m}$ luminosity for the DustPedia sample, whereas the EAGLE galaxies continue to lie along a fairly well-defined sequence. We revisit this analysis in Section 4, where we split each sample based on the galaxy sSFR.

In Fig. 8, we consider the same and the analogous scaling relations as those of Camps et al. (2016) used to calibrate the free parameters in the post-processing procedure (see Section 2.2): $M_{\text {dust }} / M_{\text {star }}$ versus stellar mass, and $M_{\text {dust }} / M_{\text {star }}$ versus sSFR (instead of NUV $-r$ colour). We decided to compare physical properties, since they are derived in the same way, and NUV $-r$ colour is generally assumed to be a proxy for sSFR (Salim et al. 2005, 2007; Schiminovich et al. 2007). We investigate whether these scaling relations are still valid considering that the samples of both observed and simulated galaxies are now larger, that all properties are derived in a self-consistent way and the post-processing procedure on the EAGLE galaxies is slightly modified, as explained in Section 2.2.

The left-hand panel of Fig. 8 shows the relation between specific dust mass and stellar mass. The figure indicates overall agreement, although some discrepancies are present. First, the large scatter found for the DustPedia sample is absent for both EAGLE samples. The reason is twofold. First, observational limitations are not accounted for. Secondly, the majority of the scattered DustPedia
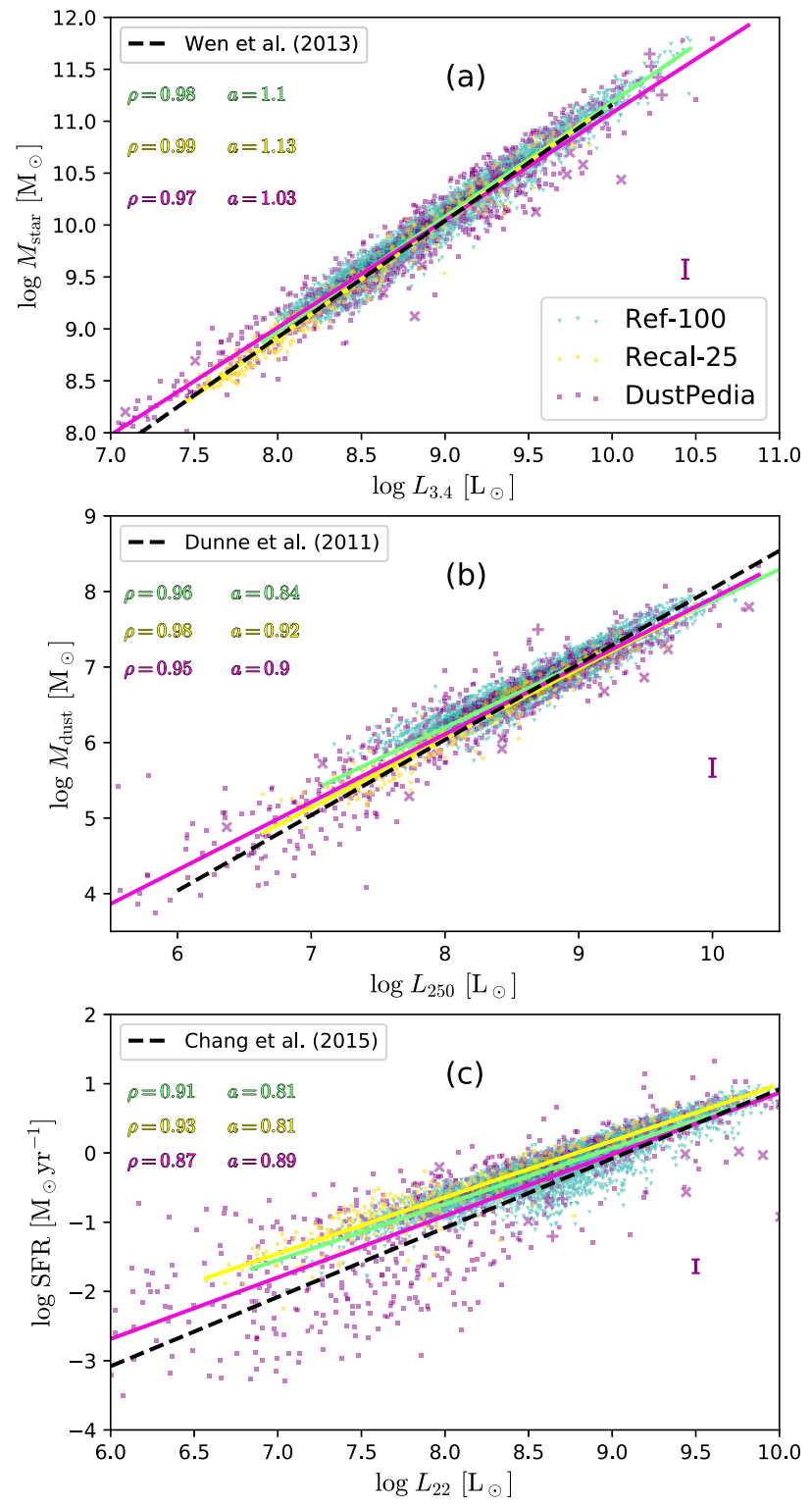

Figure 7. Luminosity proxies and appropriate properties. (a) Stellar mass versus WISE $3.4 \mu \mathrm{m}$ band, (b) dust mass versus SPIRE $250 \mu \mathrm{m}$ band, and (c) SFR versus WISE $22 \mu \mathrm{m}$ band. All physical properties are inferred from CIGALE. Coloured lines represent linear fits and their length indicates the sample domain. The dashed black line is the fit from the literature. The error bar in each panel represents the median error for the DustPedia sample. Spearman coefficients $\rho$, and slopes $a$ for each relation and data set are indicated as well. Galaxies with an AGN (strong jet) are marked with a ' $x$ ' $('+$ ').

galaxies are either low stellar mass galaxies with high sSFR that are too metal-poor to have formed much dust (De Vis et al. 2019), or early types with very little dust. Both populations are missing in the EAGLE samples due to the stellar and the dust mass thresholds, respectively. Additionally, a companion DustPedia observational paper (Casasola et al. 2020) studies the same relation focused on the late-type galaxies, showing indeed, less dispersion. The relation for EAGLE is flatter than that of the DustPedia galaxies, as also noticed by Camps et al. (2016), comparing to the HRS sample. The median dust-to-stellar mass ratio of the high-resolution Recal-25 run is systematically lower than that of Ref-100 (average difference 

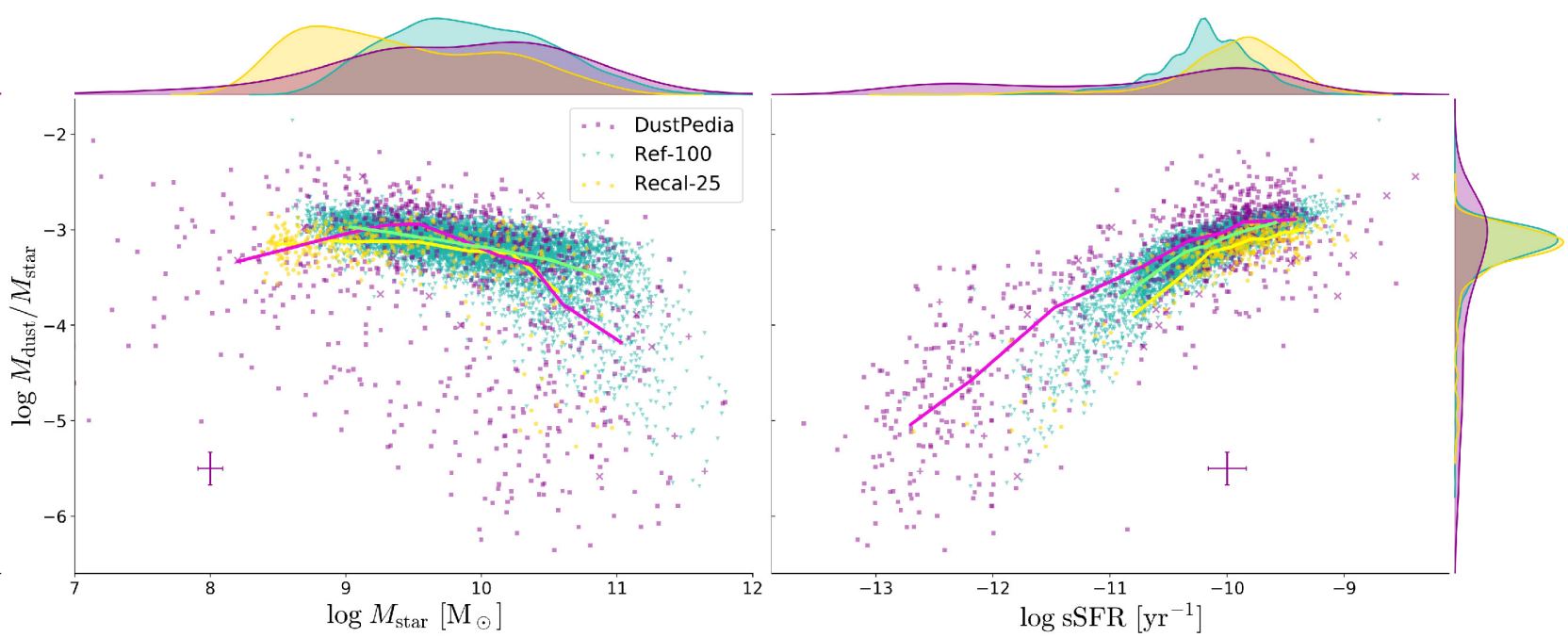

Figure 8. Scatter plots represent relations between dust-to-stellar mass ratio versus stellar mass (left) and sSFR (right). All properties are inferred from CIGALE. On the top and right normalized distributions of each property are shown. The coloured curves indicate running median. The error bars correspond to the medians of the errors for the DustPedia sample. Galaxies with an AGN (strong jet) are marked with a ' $x$ ' (' + ').

in the overlapping bins is $0.1 \mathrm{dex}$ ). This is expected since Recal-25 has a lower dust detection limit, allowing for less dusty galaxies to make the selection criterion.

The right-hand panel of Fig. 8 represents a relation between the specific dust mass and the sSFR. This relation is analogous to the one in the bottom right panel of Fig. 5. Here, we also have DustPedia galaxies evenly distributed over a large sSFR range, while the EAGLE galaxies, due to our selection effect, are mostly clustered in the high sSFR region. Galaxies from the Recal-25 sample on average have the highest SSFR ( 0.2 dex more than Ref100 , and 0.4 dex more than DustPedia in the overlapping bins). The difference in the sSFR between the EAGLE samples can be a consequence of a higher intrinsic sSFR of Recal-25, noticed in previous studies (e.g. Schaye et al. 2015; Furlong et al. 2015). At the same time, these studies also find that the EAGLE sSFRs generally tend to slightly underestimate observations, contrary to our results (see also Fig. 5, top left panel). However, they concentrate on the star-forming galaxies and our approach has an important advantage of using the same methods of deriving physical properties and their fits, for all three samples.

Additional discrepancy is that the median line of the DustPedia sample in the right plot is systematically higher than for the EAGLE samples, which is not observed in the Camps et al. (2016) study with the NUV $-r$ colour. This inconsistency can be caused by the difference in methods of acquiring properties between this study and that of Camps et al. (2016). We expect more accurate results with our method, which uses the complete galaxy SED, while they incorporated only the limited number of bands.

Another important property of a galaxy is the amount of energy absorbed by dust. It is defined as the ratio between the dust luminosity and the bolometric luminosity:

$f_{\text {abs }}=\frac{L_{\text {dust }}}{L_{\text {bolo }}}$

This ratio contains information on how optically thick a galaxy is, which further depends on the amount, composition, and geometry of dust in a galaxy. Previously, studies reported the average value of $f_{\text {abs }}$ to be $0.25 \pm 0.05$ with the highest value for late-type galaxies (and

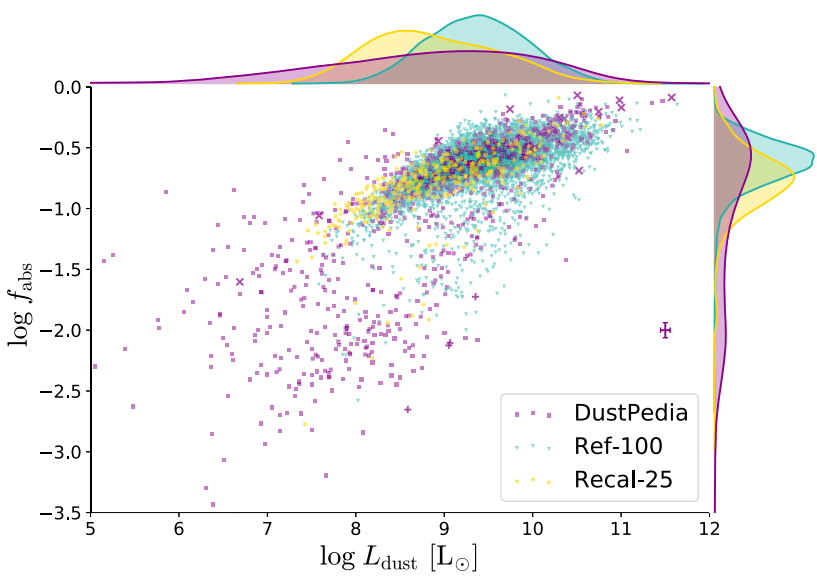

Figure 9. Amount of energy absorbed by dust versus dust luminosity, as inferred from CIGALE. The error bar corresponds to the median of the errors for the DustPedia sample. Galaxies with an AGN (strong jet) are marked with a ' $x$ ' ('+').

references therein Davies et al. 2012; Viaene et al. 2016; Bianchi et al. 2018).

The most extensive study of $f_{\text {abs }}$ is by Bianchi et al. (2018), where they used CIGALE to investigate the extent to which dust affect the stellar light in the DustPedia sample, and its correlation with different galaxy properties. They find weak trends with morphological type and some physical properties (e.g. $M_{\text {star }}$ and sSFR), and a moderate correlation with bolometric and dust luminosity for the late-type sub-sample. We repeated the same methodology for the EAGLE galaxies. The results of this consistent comparison are shown in Fig. 9, where we compare $f_{\text {abs }}$ with dust luminosity. Most of the EAGLE galaxies occupy the higher $f_{\text {abs }}$ area, indicating that most of the simulated galaxy sample has enough dust to reprocess notable amounts of stellar light. The DustPedia galaxies with the highest $f_{\text {abs }}$ are those with the AGN flag, however, not all of them have $f_{\text {abs }}$ that high (Bianchi et al. 2018). All three samples show two streams that connect around $\log L_{\text {dust }}\left[\mathrm{L}_{\odot}\right]=10$ and $\log f_{\text {abs }}=-1$. In Section 4.1, we will tackle in detail the differences in the scatter. 

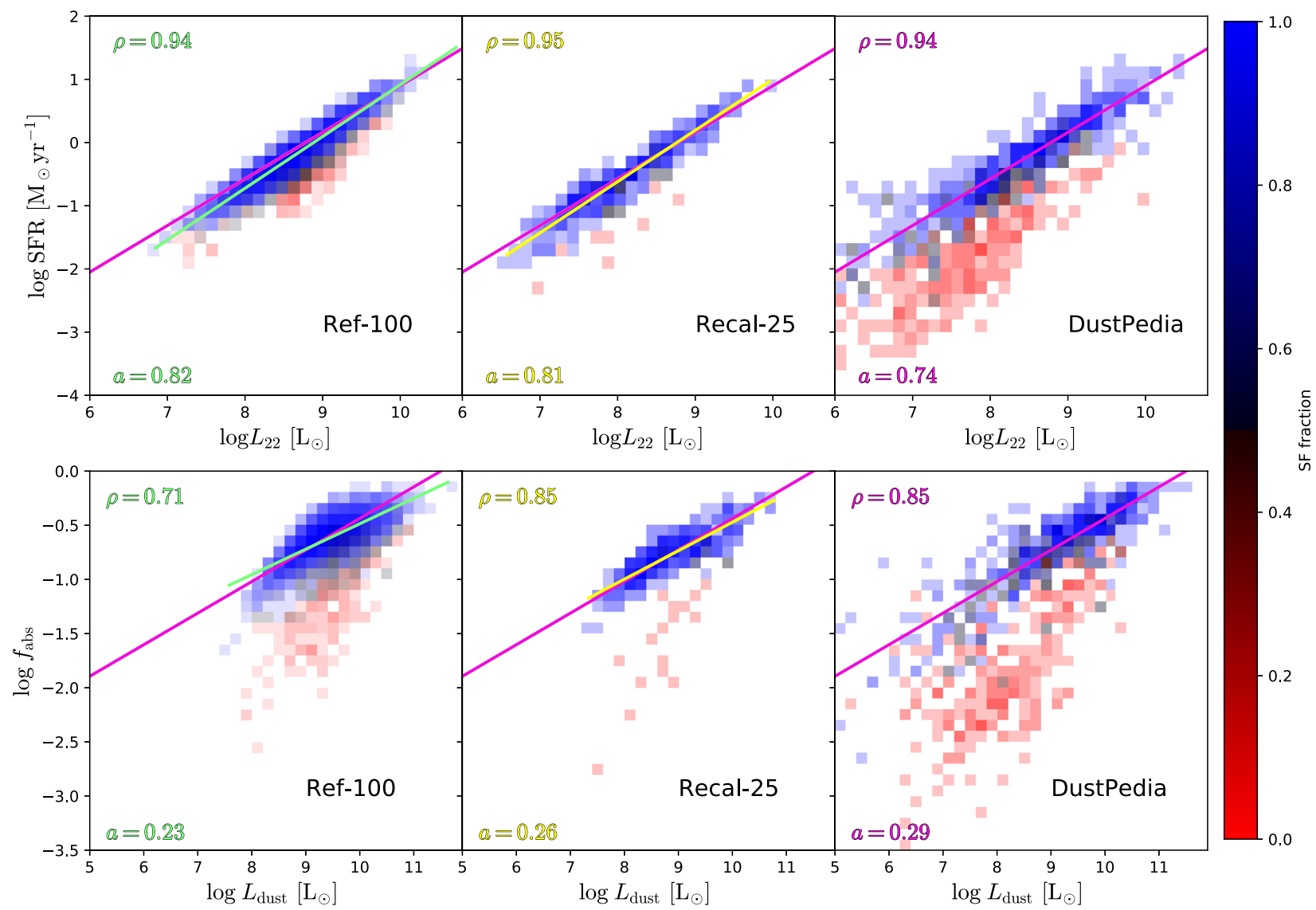

Figure 10. Top: Same as Fig. 7c with each sample in the different panel. Bins are colour-coded by the fraction of the star-forming galaxies in the bin. Magenta line represents a fit only through star-forming DustPedia galaxies. Green and yellow lines indicate fits of the EAGLE run in the appropriate panel, again only for the star-forming galaxies. Spearman coefficients $\rho$, and slopes $a$ for each data set are indicated in the top left corner. Transparency indicates the number of galaxies in each bin. Bottom: Same as Fig. 9 with each sample in the different panel, analogue to the top row.

\section{DISCUSSION}

The results from this study so far indicate overall agreement between simulations and observations, apart from a few discrepancies, mostly differences in the scatter and offsets in the relations and the SED regimes associated with SFR. This confirms earlier findings of Baes et al. (2019), who reported small but systematic tensions in certain sections of the cosmic SED. In general, these may arise from: a different galaxy population mix in the three samples, and/or differences in the stellar/dust properties of EAGLE and DustPedia, coming from the imperfections of the post-processing procedure or limitations in EAGLE recipes for galaxy formation. In the following sections, we will address each of these possible causes for deviations.

\subsection{The galaxy population}

As discussed in Section 3.3, relations between different galaxy properties and appropriate luminosity proxies for all three samples are reasonably tight and in agreement, except the relation between WISE $22 \mu \mathrm{m}$ luminosity and SFR, where the discrepancies between the three samples are higher (see Fig. 7c). To better understand the differences between the three galaxy samples, we further analyse this relation. The top row of Fig. 10 represents the same as Fig. 7c with the different samples in the different panels. the colour-coding is based on the fraction of star-forming galaxies in each bin. We assume a galaxy is star-forming if its sSFR satisfies log sSFR > $-10.8 \mathrm{yr}^{-1}$ (Salim 2014). This figure clearly indicates that the discrepancy in the SFR $-L_{22}$ relation between the three samples is primarily due to a different galaxy mix in the samples. For all three, star-forming galaxies form the same tight sequence. The main distinction is the large fraction of galaxies in the DustPedia sample with WISE $22 \mu \mathrm{m}$ radiation that does not solely relates to the star formation activity, but that is mostly arising from the evolved stars or the warm dust heated by them (Madden, Vigroux \& Sauvage 1999; Xilouris et al. 2004; Simonian \& Martini 2017). These quiescent galaxies are removed from the EAGLE-SKIRT sample, since they lack sufficient amounts of dust to make our selection criterion. Accordingly, when we consider only star-forming galaxies (i.e. galaxies in the blue bins), and compare to Fig. 7c, the scatter is largely reduced: the Spearman coefficients are now almost the same for all three samples, and the gradient of the power-law fit for DustPedia is lower than the one for the full sample, agreeing better with the simulations.

In Section 3.3, we demonstrated that the global dust emission properties, represented by the $f_{\text {abs }}$ versus $L_{\text {dust }}$ relation, agree for the three samples, apart from the region of low dust emission (see Fig. 9). Now, following the same approach as for the SFR $-L_{22}$ relation, we inspect if the differences in the galaxy populations are again driving the tension between the three samples. In the bottom row of Fig. 10, we compare $f_{\text {abs }}$ with dust luminosity. The samples are in the different panels, colour-coded by fraction of star-forming 


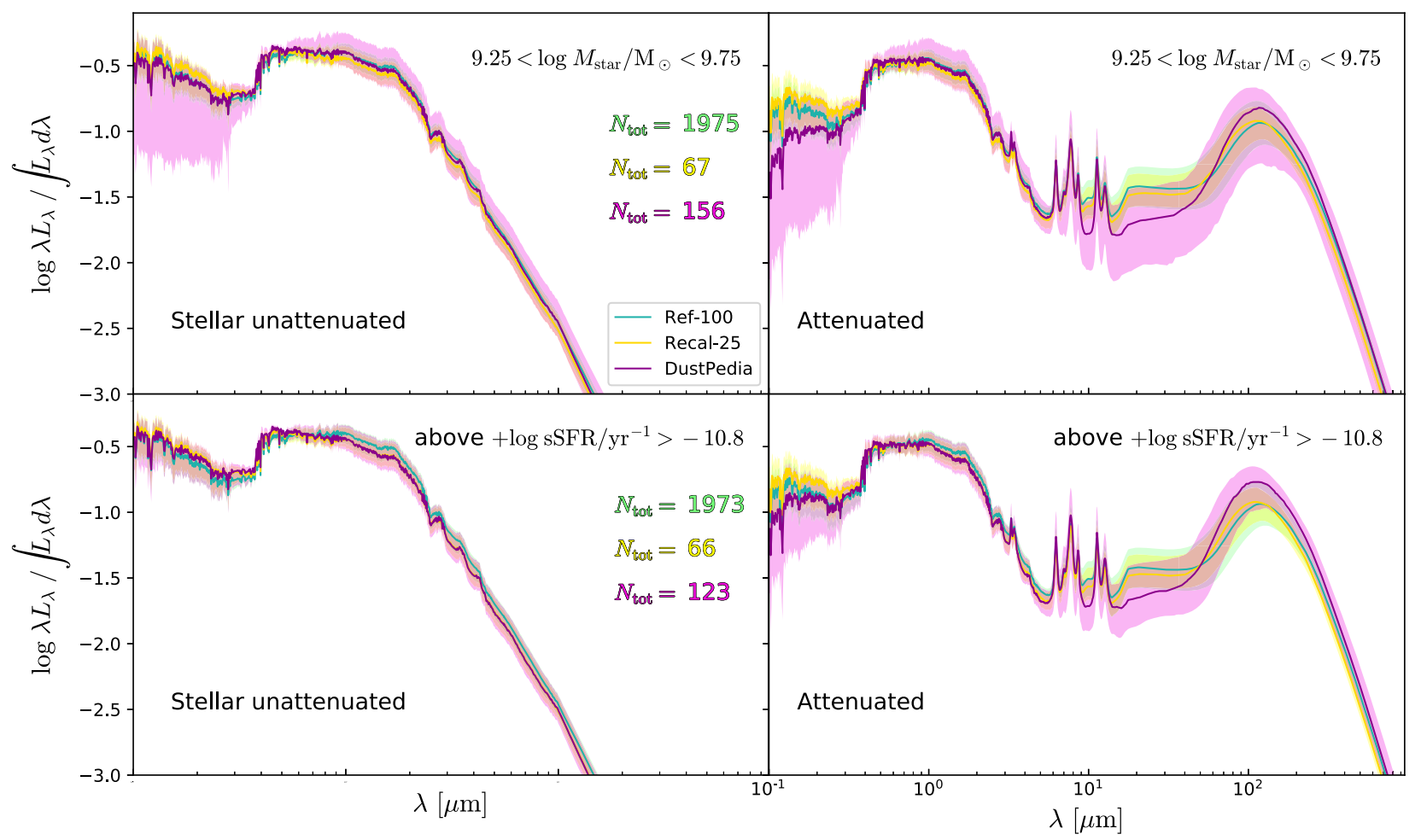

Figure 11. Similar to Fig. 6 with the top row including only galaxies in the indicated stellar mass range while the bottom row excludes also the passive galaxies.

galaxies in each bin. It is apparent from the figure that the differences in the galaxy population mix affect the relation notably: when we neglect the dust-poor galaxies, the results become comparable. For instance, although Recal-25 has a lower average value of $f_{\text {abs }}$ (as already seen from the distribution in Fig. 9 for the whole sample), star-forming galaxies from this sample follow a hardly distinguishable trend from the one of DustPedia. In comparison, a slight offset is seen for the Ref-100 sample, which is expected considering the Recal-25 run has a higher resolution and better sampled discs (Trayford et al. 2017). For a galaxy property that depends on the geometry of a galaxy, such as $f_{\text {abs }}$ (Viaene et al. 2016; Bianchi et al. 2018), a sample with more realistic spiral galaxies will reach a better agreement with observations.

Based on Fig. 10, we are inclined to conclude that differences in the galaxy population between the samples can explain the tensions in the relations discussed in Section 3. However, these differences alone cannot explain the substantial offset between EAGLE and DustPedia along the whole range of $L_{22} / L_{3.4}$ luminosity ratios seen in Fig. 5 bottom right. To understand how strong the effect of the different sample selection actually is, we examine the median SEDs again. We select galaxies in the small mass range of $9.25<$ $\log M_{\text {star }} / \mathrm{M}_{\odot}<9.75$ (based on the CIGALE results), and reanalyse the features of their SEDs. Fig. 11 is analogous to Fig. 6, with the top row including only galaxies in the selected stellar mass range. In this range, most of the galaxies are late type with a significant amount of dust, which corresponds better to the EAGLE sample. There is a clearly better agreement between DustPedia and EAGLE, but the discrepancies remain in both the UV and MIR part of the galaxy spectrum. In the attenuated FUV band, the median DustPediaRef-100 (DustPedia-Recal-25) difference is 0.19 dex (0.27 dex).
For the WISE $22 \mu \mathrm{m}$ band, the difference is 0.25 dex $(0.21 \mathrm{dex}$, same as when comparing full samples). In later sections we discuss discrepancies in these bands for a range of mass bins.

For a fixed $M_{\text {star }}$, the DustPedia sample contains both star-forming and passive early-type galaxies (in this bin, $\approx 21$ per cent are earlytype galaxies). For a direct comparison with the EAGLE sample, we thus also included an additional constraint: $\log \mathrm{SSFR}>-10.8 \mathrm{yr}^{-1}$, since galaxies with a lower sSFR are mostly passive. The new constraint minimally affects the EAGLE-SKIRT sample, because almost all galaxies in the selected mass bin already have the sSFRs above $-10.8 \mathrm{yr}^{-1}$. This is in agreement with Katsianis et al. (2019), who found the fraction of only $\approx 0.1$ early-type galaxies at similar $M_{\text {star }}$ and sSFRs, for the whole EAGLE reference simulation, at $z=$ 0 . The results are shown in bottom panels of Fig. 11. Showing the unattenuated stellar light only, the left-hand panel shows an almost perfect agreement. The discrepancies in the attenuated SEDs are smaller, but still present: in FUV band the difference for DustPediaRef-100 (DustPedia-Recal-25) is 0.08 (0.16), and in WISE $22 \mu \mathrm{m}$ band the difference for DustPedia-Ref-100 (DustPedia-Recal-25) is $0.21(0.16)$ dex. The average difference in the FIR seems to have increased. Here, we can expect that the differences in the dust models [by Zubko et al. (2004) in the post-processing, and THEMIS in the SED fitting] have an effect (see Section 2.4). The prominent features around $20 \mu \mathrm{m}$ modelled by Zubko et al. (2004), and then fitted by THEMIS would produce an excess at these wavelengths, as the model would try to fit PAH features with the continuum emission.

The findings of this section confirm that the differences in the galaxy populations between the three samples greatly influence, yet cannot fully explain the differences seen in the scaling relations and the SEDs. 


\subsection{The EAGLE simulations and the SKIRT post-processing}

We now turn to another possible cause for the differences between simulations and observations: the limitations of the EAGLE simulations and the procedure applied to the EAGLE particle data, in order to incorporate dust in the simulations (see Section 2.2).

A growing number of studies have analysed the EAGLE simulations and how well they reproduce different observables that were not used for their calibration (e.g. Crain et al. 2015; Furlong et al. 2015; Lagos et al. 2015; Schaye et al. 2015; Trayford et al. 2015, 2016; Furlong et al. 2017; Katsianis et al. 2017; Tescari et al. 2018). Considering the good agreement on the stellar emission only (Fig. 11, left-hand panels), we also conclude that the stellar properties of the EAGLE galaxies are representative of the stellar properties of our sample of real galaxies. Additionally, as explained in Section 2.4, we performed a check where we compare the values of intrinsic properties of EAGLE with the values derived from CIGALE and we find that the data are in agreement.

In the remainder of this section, we focus on the impact of our post-processing recipe on the results. During the calibration of the parameters in the post-processing, in the IR domain, only SPIRE bands were used (Camps et al. 2016). This implies that the MIRFIR regime of the galaxy spectrum is essentially unconstrained and susceptible to discrepancies. In Section 3.2, we have already seen that at these wavelengths the median SEDs are discrepant, even if we limit the analysis to a specific stellar mass and SSFR bin (Fig. 11). Thus, it may be assumed that they are caused by the characteristic treatment of the star-forming regions, applied using the MAPPINGS-III templates.

To investigate this further, we analyse a galaxy scaling relation based on the UV and dust emission. To lessen the effect of the different galaxy populations in each sample, we concentrate only on the star-forming galaxies, i.e. galaxies with $\log \mathrm{SSFR}>-10.8 \mathrm{yr}^{-1}$. We analyse the IRX $-\beta$ relation presented by Meurer, Heckman \& Calzetti (1999), where IRX is the infrared excess defined as

$\mathrm{IRX}=\log \frac{L_{\mathrm{dust}}}{L_{\mathrm{FUV}}}$,

while $\beta$ is the UV slope defined as

$\beta=\frac{\log f_{\nu(\mathrm{NUV})} / f_{\nu(\mathrm{FUV})}}{\log \lambda_{\mathrm{NUV}} / \lambda_{\mathrm{FUV}}}-2$,

where $f_{v}$ is the flux density. The relation, considering it is sensitive to the dust attenuation, has been thoroughly analysed for a wide range of redshifts (e.g. Meurer et al. 1999; Kong et al. 2004; Overzier et al. 2011; Boquien et al. 2012; Salim \& Boquien 2019). The relation demonstrates that star-bursting galaxies have redder UV (towards positive $\beta$ values) colour if more UV radiation is reprocessed by dust.

Fig. 12 shows the IRX $-\beta$ relation for star-forming galaxies in all three samples. All properties needed to calculate IRX and $\beta$ are derived using CIGALE. Dashed lines are fits for star-bursting galaxies from Overzier et al. (2011) and Boquien et al. (2012). Remarkably, the EAGLE SEDs fits agree well within the range of these observational relations, comparable to the previous studies that included cosmological simulations (e.g. Narayanan et al. 2018a; Hou et al. 2019). In general, star-forming EAGLE galaxies show the same trend as star-forming DustPedia galaxies. However, Ref-100 shows a small but systematic offset that can be partially attributed to resolution and the fact that Recal-25 has a higher UV output (since it has on average higher sSFR, Schaye et al. 2015) and hence a smaller IRX.

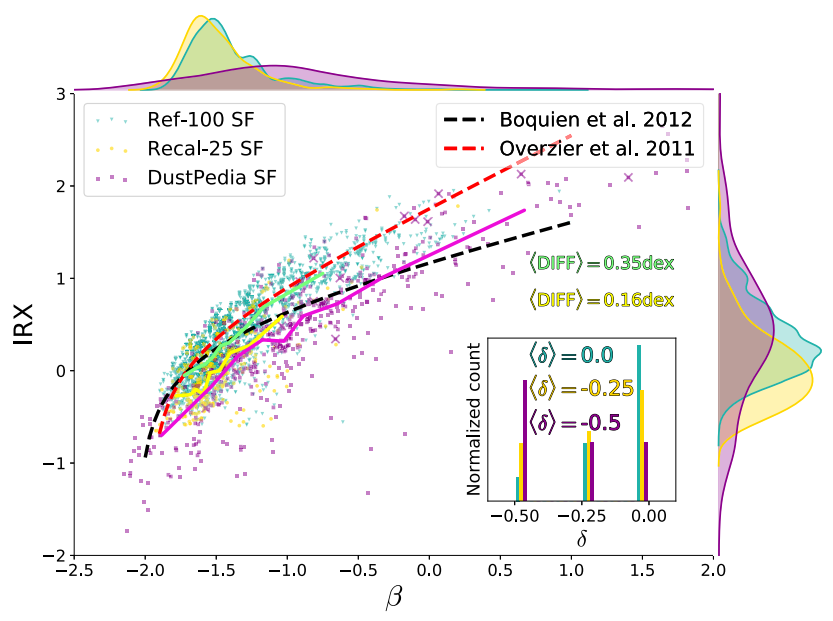

Figure 12. IR excess versus UV slope, only for the star-forming subsamples. The dashed lines represent fits from the literature, solid lines are the running medians. The number in green (yellow) represents the average deviation of the Ref-100 (Recal-25) from the DustPedia running median. The inset shows the distribution of the attenuation curve slopes, as inferred from CIGALE.

Another origin of the discrepancy comes from the difference in the attenuation curve. As mentioned in Section 2.4, our CIGALE models include a modified Calzetti et al. (2000) attenuation curve, implemented with a free parameter slope and without a UV bump (Nersesian et al. 2019). The distribution of slopes for all samples is shown in the inset of Fig. 12, where the DustPedia sample has the steepest median slope and Ref-100 the shallowest. Salim \& Boquien (2019) analysed IRX $-\beta$ relation for around 23000 lowredshift galaxies from the GSWLC-2 sample (Salim, Boquien \& Lee 2018) and argue that the scatter and the offset from the Overzier et al. (2011) curve are driven by the diversity of the attenuation curves, mainly their slopes. They demonstrate ${ }^{8}$ that the shallower the slope is, the higher the galaxy is in the IRX $-\beta$ plot, which is reproduced in our study.

The offset between the median lines, seen in the IRX $-\beta$ relation, is caused by the offset in the median slope of the attenuation curve. Narayanan et al. (2018b) investigated the main influence on the diversity of the slopes on a sample of 'zoom-in' simulated galaxies and they highlight the importance of the star-to-dust geometry, i.e. high fraction of obscured young and low fraction of obscured old stars steepen the attenuation curve. Interpreting results in this context, we argue that our sub-scale modelling of the $\mathrm{H}$ II regions in our EAGLE-SKIRT post-processing algorithm can be improved, possibly by changing the value of $f_{\mathrm{PDR}}$. We note that Trayford et al. (2020), analysing the slope of the attenuation curves of the EAGLE galaxies, found that Recal-25 does not have the steepest slope, contrary to our result. However, they inspected only the diffuse dust while the effect of the birth clouds will steepen the curve for the galaxies with more star formation, since the spectrum of the young, blue stars will be greatly reddened. Recal-25 has intrinsically higher sSFR than Ref-100 which drives the slope towards steeper values. Additionally, their galaxy sample has a different stellar, dust mass and redshift thresholds, and they calculated the attenuation at a fixed dust surface density. Furthermore, to calculate the attenuation, Trayford et al. (2020) used two different SKIRT runs (with and

\footnotetext{
${ }^{8}$ Although using non-zero UV bump strength.
} 
without diffuse dust), while we use CIGALE derived results on one SKIRT run.

\subsection{Towards an improved post-processing recipe}

Taken together, the results from this study indicate that the derivation of fluxes (in a number of bands), therefore SEDs, and consequently physical properties of the EAGLE galaxies, could be improved. In principle, we could repeat the calibration exercise by Camps et al. (2016) and search the entire space of the subgrid parameters for the combination that minimizes the tension between EAGLE and DustPedia, using the relations presented in this paper. However, such a wide search is a daunting task, given the number of possible free parameters in the subgrid recipe, and the computational cost to run radiative transfer simulations for the EAGLE galaxies, even at $z=0$. Instead, we can use the observed differences to guide us in which direction the post-processing calibration should be heading. Concretely, we investigate whether the flux differences are correlated with any particular physical properties, and whether these correlations uncover additional effects of the post-processing procedure and its calibration.

We compute the ratio of luminosity in a band for the EAGLE samples to luminosity in the same band for the DustPedia sample, in narrow 2D bins of $M_{\text {star }}$ and sSFR or of specific dust mass and $M_{\text {star }}$. The binning minimizes the effect of the different galaxy mixture in the different samples. We use the luminosity values from the CIGALE fits for all three samples and again we exclude 10 per cent of the galaxies with the most irregular SEDs. We have investigated differences in different bands, from FUV to $250 \mu \mathrm{m}$, and their potential correlation with stellar mass, (specific) dust mass and (specific) SFR, however we only show those that reveal clear trends. The results are presented in Fig. 13. Each point in the specific dust mass bin (top panel) is a median of those in different $M_{\text {star }}$ bins. Each point in the stellar mass bin (middle and bottom panels) is a median of those in different sSFR bins.

From the top panel, it is evident that deviations in the SPIRE $250 \mu \mathrm{m}$ band correlate with the specific dust mass. The decreasing slope indicates that for the low specific dust mass galaxies our models overpredict radiation in the SPIRE $250 \mu \mathrm{m}$ band and contrarily, the model slightly underpredicts the $250 \mu \mathrm{m}$ luminosity for the very dusty galaxies. This discrepancy may be a symptom of the constant dust-to-metal ratio we assume for all EAGLE galaxies (see Section 2.2), since the diffuse dust dominates at these wavelengths. In particular, it is evident that simply increasing (decreasing) the global dust-to-metal ratio will not eliminate the difference in the slope of the lines in the figure. Recent studies report correlations of the dust-to-metal ratio with galaxy properties like stellar mass and metallicity (De Vis et al. 2019; Li, Narayanan \& Davé 2019; Lagos et al. 2019), however it remains to be seen if these correlations are strong enough in the stellar mass regime we consider here. Nevertheless, as the dust from the birth clouds can also contribute at these wavelengths, we argue that a modification of the implementation of the subgrid star-forming regions is required as well.

We detected opposite trends with $M_{\text {star }}$ for luminosities in the FUV and the WISE $22 \mu \mathrm{m}$ bands, as shown in Fig. 13, bottom two panels. The correlation is stronger for Recal-25 than for the lower resolution Ref-100. These trends reveal that the FUV and MIR emissions are coupled, which is expected since more attenuation of the stellar light in the FUV implies more dust emission in the MIR. Interestingly, neither Fig. 6 nor Fig. 11 unveils this connection - the figures only indicate an excess in both parts of the spectrum. Ideally, the changes in representation of the star-
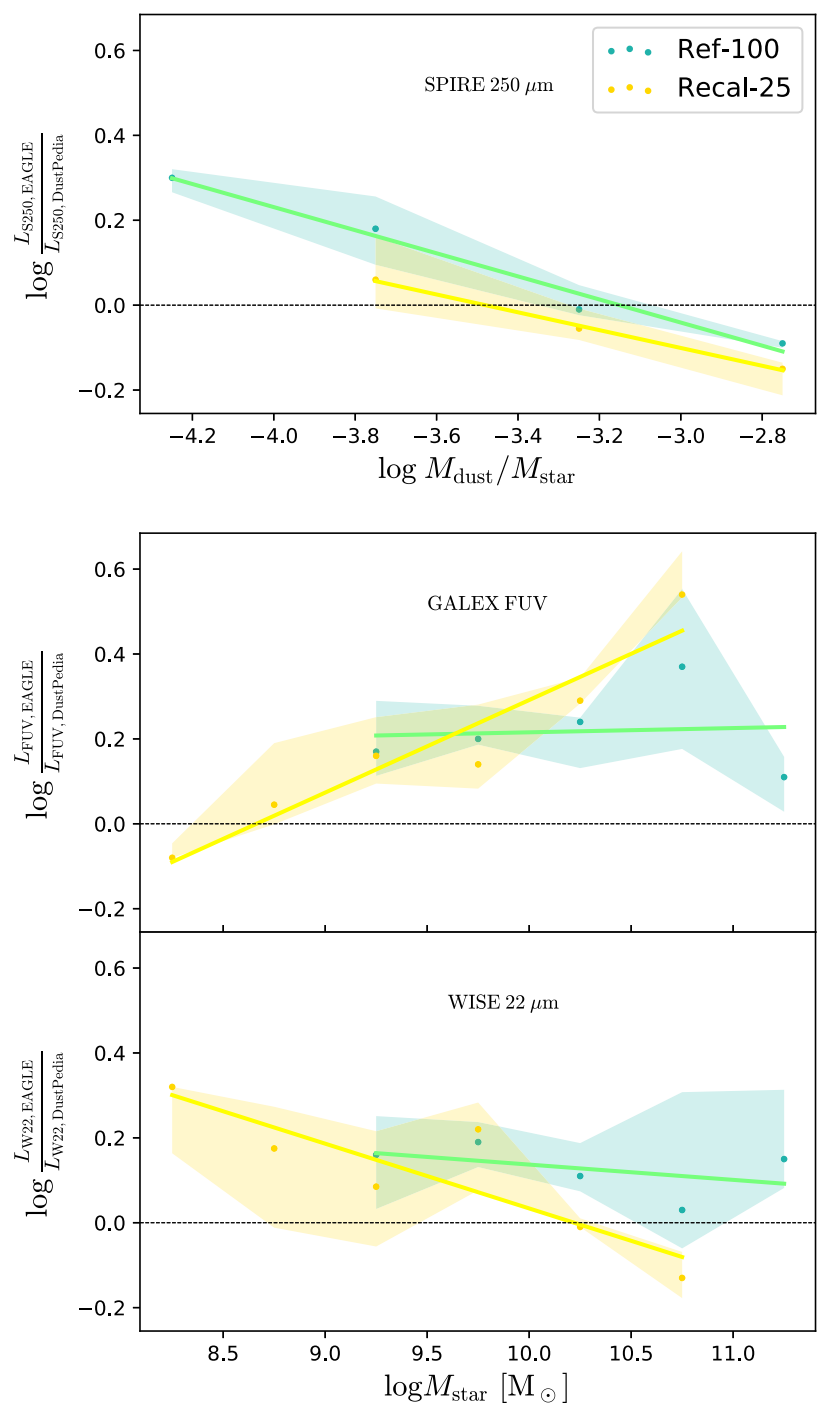

Figure 13. Top: Differences in the SPIRE $250 \mu \mathrm{m}$ band between EAGLE and DustPedia as a function of the specific dust mass ( $x$-axis) and the stellar mass (weight in each specific dust mass bin). Middle: Same as top but for the FUV band and as a function of the stellar mass and sSFR. Bottom: Similar as above but for the WISE $22 \mu \mathrm{m}$ band. The lines represent the linear fit, the shaded regions show $16-84$ per cent range.

forming regions should be such that, in the lowest mass galaxies, the FUV emission remains unchanged whereas the MIR emission decreases by $\sim 0.3$ dex. For the most massive galaxies, the FUV attenuation should increase substantially, without a major change in the MIR emission. Because the FUV emission predominantly originates from young and massive stars and WISE $22 \mu \mathrm{m}$ from dust heated by these stars, these bands are severely affected by the geometry of the star-forming regions. Improvement would be expected if the most massive stars in the star-forming regions would have a higher $f_{\mathrm{PDR}}$, and vice versa for the less massive. Whether this adjustment of the geometry is possible with MAPPINGS-III templates, it is not clear at this stage.

Our analysis demonstrates that further study on the modelling of the star-forming regions is needed. There are several approaches that can be taken to tackle this problem. The use of the MAPPINGSIII templates can be revisited, however with improvement of the original procedure by applying additional constraints based on the 
results from this study. For instance, the dust-to-metal fraction, currently a constant parameter in the procedure, can be modified to a variable one. We will explore these avenues in future work. The forthcoming cosmological simulations can benefit from these types of post-processing procedures; they can improve the calibration of the subgrid parameters of the simulations, since the comparison with the observations could then be implemented directly in the observational, i.e. flux, space.

\section{SUMMARY}

In this study, we investigated across a broad wavelength range whether the EAGLE simulations combined with SKIRT, provide a proper description of galaxies in the Local Universe. We compare them with the DustPedia sample in a consistent manner using the SED fitting tool CIGALE to derive all physical properties for all the samples. Though galaxies have very different distributions in individual quantities, the relations between these quantities broadly hold, but a number of discrepancies are present as well:

(i) Comparing scaling relations between luminosities directly from the data bases, reveals discrepancies in the MIR, with much better agreement in the optical and FIR range. Similar results are obtained comparing fitted SEDs and the physical propertyluminosity proxy relations, with additional finding of deviations in the UV range.

(ii) Scaling relations between the physical properties show that those relations that are dependent on the global energy, diffuse dust and stellar mass show satisfactory agreement, while the relations and SED regimes primarily driven by the properties of the starforming regions show discordance.

(iii) To understand the origin of these discrepancies, we analyse only the star-forming galaxies, applying the sSFR $>-10.8 \mathrm{yr}^{-1}$ threshold. Most of the relations improved significantly, indicating the importance of the difference in the galaxy population mix between the samples.

(iv) An analysis of the IRX $-\beta$ relation, despite the great overall agreement, shows discrepancies which are mainly caused by the limitations in the subgrid treatment of the star-forming regions.

(v) We quantify the deviations in the median SEDs, and their correlation with galaxy properties. We find trends that can help to improve and optimize the future re-calibration process, necessary for the more realistic modelling of the HII regions.

(vi) This detailed comparison highlights the successes and shortcomings of the current panchromatic modelling. This new knowledge indicates the areas where the procedure can be improved, with the aim to implement it in the future cosmological simulations to assist in their calibration process.

\section{ACKNOWLEDGEMENTS}

DustPedia is a collaborative focused research project supported by the European Union under the Seventh Framework Programme (2007-2013) call (proposal no. 606847). We acknowledge the Virgo Consortium for making EAGLE simulation data available.

This research made extensive use of the NUMPY, MATPLOTLIB, and PANDAS PYTHON packages.

\section{REFERENCES}

Abadi M. G., Navarro J. F., Steinmetz M., Eke V. R., 2003, ApJ, 597, 21 Andrews S. K. et al., 2017, MNRAS, 470, 1342
Aoyama S., Hou K.-C., Hirashita H., Nagamine K., Shimizu I., 2018, MNRAS, 478, 4905

Aoyama S. et al., 2019, MNRAS, 484, 1852

Baes M. et al., 2003, MNRAS, 343, 1081

Baes M., Verstappen J., De Looze I., Fritz J., Saftly W., Vidal Pérez E., Stalevski M., Valcke S., 2011, ApJS, 196, 22

Baes M., Trčka A., Camps P., Nersesian A., Trayford J., Theuns T., Dobbels W., 2019, MNRAS, 484, 4069

Bahé Y. M. et al., 2017, MNRAS, 470, 4186

Bauer A. E. et al., 2013, MNRAS, 434, 209

Bell E. F., McIntosh D. H., Katz N., Weinberg M. D., 2003, ApJS, 149, 289

Bianchi S. et al., 2018, A\&A, 620, A112

Bignone L. A., Pedrosa S. E., Trayford J. W., Tissera P. B., Pellizza L. J., 2020, MNRAS, 491, 3624

Boquien M. et al., 2012, A\&A, 539, A145

Boquien M. et al., 2016, A\&A, 591, A6

Boquien M., Burgarella D., Roehlly Y., Buat V., Ciesla L., Corre D., Inoue A. K., Salas H., 2019, A\&A, 622, A103

Boselli A. et al., 2010, PASP, 122, 261

Brinchmann J., Charlot S., Kauffmann G., Heckman T., White S. D. M., Tremonti C., 2013, MNRAS, 432, 2112

Bruzual G., Charlot S., 2003, MNRAS, 344, 1000

Calzetti D., Kinney A. L., Storchi-Bergmann T., 1994, ApJ, 429, 582

Calzetti D., Armus L., Bohlin R. C., Kinney A. L., Koornneef J., StorchiBergmann T., 2000, ApJ, 533, 682

Calzetti D. et al., 2010, ApJ, 714, 1256

Camps P., Baes M., 2015, Astron. Comput., 9, 20

Camps P., Trayford J. W., Baes M., Theuns T., Schaller M., Schaye J., 2016, MNRAS, 462, 1057

Camps P. et al., 2018, ApJS, 234, 20

Casasola V. et al., 2017, A\&A, 605, A18

Casasola V. et al., 2020, A\&A, 633, A100

Chabrier G., 2003, PASP, 115, 763

Chang Y.-Y., van der Wel A., da Cunha E., Rix H.-W., 2015, ApJS, 219, 8

Ciesla L. et al., 2016, A\&A, 585, A43

Clark C. J. R. et al., 2018, A\&A, 609, A37

Cluver M. E., Jarrett T. H., Dale D. A., Smith J.-D. T., August T., Brown M. J. I., 2017, ApJ, 850, 68

Cochrane R. K. et al., 2019, MNRAS, 488, 1779

Cortese L. et al., 2012, A\&A, 540, A52

Crain R. A. et al., 2015, MNRAS, 450, 1937

Crain R. A. et al., 2017, MNRAS, 464, 4204

Davé R., Anglés-Alcázar D., Narayanan D., Li Q., Rafieferantsoa M. H., Appleby S., 2019, MNRAS, 486, 2827

Davies J. I. et al., 2012, MNRAS, 419, 3505

Davies J. I. et al., 2017, PASP, 129, 44102

De Vis P. et al., 2019, A\&A, 623, A5

Dickinson H. et al., 2018, ApJ, 853, 194

Diemer B. et al., 2019, MNRAS, 487, 1529

Dobbels W. et al., 2020, A\&A, 634, A57

Driver S. P. et al., 2011, MNRAS, 413, 971

Dunne L. et al., 2011, MNRAS, 417, 1510

Dwek E., 1998, ApJ, 501, 643

Eales S. et al., 2012, ApJ, 761, 168

Furlong M. et al., 2015, MNRAS, 450, 4486

Furlong M. et al., 2017, MNRAS, 465, 722

Galliano F., Galametz M., Jones A. P., 2018, ARA\&A, 56, 673

Griffin M. J. et al., 2010, A\&A, 518, L3

Groves B., Dopita M., Sutherland R., Kewley L., Fischera J., Leitherer C., Brandl B., van Breugal W., 2008, ApJS, 176, 438

Guidi G., Scannapieco C., Walcher C. J., 2015, MNRAS, 454, 2381

Hao C.-N., Kennicutt R. C., Johnson B. D., Calzetti D., Dale D. A., Moustakas J., 2011, ApJ, 741, 124

Hou K.-C., Aoyama S., Hirashita H., Nagamine K., Shimizu I., 2019, MNRAS, 485, 1727

Hunt L. K. et al., 2019, A\&A, 621, A51

Jones A. P., Köhler M., Ysard N., Bocchio M., Verstraete L., 2017, A\&A, 602, A46 
Jonsson P., Groves B. A., Cox T. J., 2010, MNRAS, 403, 17

Katsianis A., Tescari E., Wyithe J. S. B., 2016, Publ. Astron. Soc. Aust., 33, e029

Katsianis A. et al., 2017, MNRAS, 472, 919

Katsianis A. et al., 2019, ApJ, 879, 11

Katsianis A. et al., 2020, MNRAS, 492, 5592

Kennicutt R. C., Evans N. J., 2012, ARA\&A, 50, 531

Kong X., Charlot S., Brinchmann J., Fall S. M., 2004, MNRAS, 349, 769

Lagos C. d. P. et al., 2015, MNRAS, 452, 3815

Lagos C. d. P. et al., 2019, MNRAS, 489, 4196

Lee J. C., Hwang H. S., Ko J., 2013, ApJ, 774, 62

Li Q., Narayanan D., Davé R., 2019, MNRAS, 490, 1425

Liang L., Feldmann R., Faucher-Giguère C.-A., Kereš D., Hopkins P. F., Hayward C. C., Quataert E., Scoville N. Z., 2018, MNRAS, 478, L83

Liang L. et al., 2019, MNRAS, 489, 1397

Liske J. et al., 2015, MNRAS, 452, 2087

Ma X. et al., 2019, MNRAS, 487, 1844

McAlpine S. et al., 2016, Astron. Comput., 15, 72

McKinnon R., Torrey P., Vogelsberger M., 2016, MNRAS, 457, 3775

McKinnon R., Torrey P., Vogelsberger M., Hayward C. C., Marinacci F., 2017, MNRAS, 468, 1505

Madden S. C., Vigroux L., Sauvage M., 1999, in Cox P., Kessler M., eds, ESA SP-427: The Universe as Seen by ISO. ESA, Noordwijk, p. 933

Meurer G. R., Heckman T. M., Calzetti D., 1999, ApJ, 521, 64

Mitchell P. D., Lacey C. G., Baugh C. M., Cole S., 2013, MNRAS, 435, 87

Morrissey P. et al., 2007, ApJS, 173, 682

Mullaney J. R., Alexander D. M., Goulding A. D., Hickox R. C., 2011, MNRAS, 414, 1082

Narayanan D., Davé R., Johnson B. D., Thompson R., Conroy C., Geach J., 2018a, MNRAS, 474, 1718

Narayanan D., Conroy C., Davé R., Johnson B. D., Popping G., 2018b, ApJ, 869,70

Nelson D. et al., 2018, MNRAS, 475, 624

Nersesian A. et al., 2019, A\&A, 624, A80

Neugebauer G. et al., 1984, ApJ, 278, L1

Noeske K. G. et al., 2007, ApJ, 660, L43

Noll S., Burgarella D., Giovannoli E., Buat V., Marcillac D., Muñoz-Mateos J. C., 2009, A\&A, 507, 1793

Norris M. A., Meidt S., Van de Ven G., Schinnerer E., Groves B., Querejeta M., 2014, ApJ, 797, 55

Overzier R. A. et al., 2011, ApJ, 726, L7

Pappalardo C. et al., 2016, A\&A, 589, A11

Pilbratt G. L. et al., 2010, A\&A, 518, L1

Pillepich A. et al., 2018, MNRAS, 473, 4077

Planck Collaboration I, 2011, A\&A, 536, A1

Planck Collaboration XVI, 2014, A\&A, 571, A16

Poglitsch A. et al., 2010, A\&A, 518, L2

Popescu C. C., Tuffs R. J., 2002, MNRAS, 335, L41

Rémy-Ruyer A. et al., 2014, A\&A, 563, A31

Rodriguez-Gomez V. et al., 2019, MNRAS, 483, 4140

Rosa-González D., Terlevich E., Terlevich R., 2002, MNRAS, 332, 283

Sales L. V. et al., 2015, MNRAS, 447, L6

Salim S., 2014, Serb. Astron. J., 189, 1

Salim S., Boquien M., 2019, ApJ, 872, 23

Salim S. et al., 2005, ApJ, 619, L39

Salim S. et al., 2007, ApJS, 173, 267

Salim S., Boquien M., Lee J. C., 2018, ApJ, 859, 11

Salpeter E. E., 1955, ApJ, 121, 161

Schaye J. et al., 2015, MNRAS, 446, 521

Schiminovich D. et al., 2007, ApJS, 173, 315

Simonian G. V., Martini P., 2017, MNRAS, 464, 3920

Skibba R. A. et al., 2011, ApJ, 738, 89

Skrutskie M. F. et al., 2006, AJ, 131, 1163

Springel V., 2005, MNRAS, 364, 1105

Tescari E. et al., 2018, MNRAS, 473, 380

Trayford J. W. et al., 2015, MNRAS, 452, 2879

Trayford J. W., Theuns T., Bower R. G., Crain R. A., Lagos C. d. P., Schaller M., Schaye J., 2016, MNRAS, 460, 3925
Trayford J. W. et al., 2017, MNRAS, 470, 771

Trayford J. W., Frenk C. S., Theuns T., Schaye J., Correa C., 2019, MNRAS, 483,744

Trayford J. W., Lagos C. d. P., Robotham A. S. G., Obreschkow D., 2020, MNRAS, 491, 3937

Viaene S. et al., 2016, A\&A, 586, A13

Vogelsberger M. et al., 2014, MNRAS, 444, 1518

Vogelsberger M., Marinacci F., Torrey P., Puchwein E., 2020, NatRP, 2, 42

Wen X.-Q., Wu H., Zhu Y.-N., Lam M. I., Wu C.-J., Wicker J., Zhao Y.-H., 2013, MNRAS, 433, 2946

Werner M. W. et al., 2004, ApJS, 154, 1

Wright E. L. et al., 2010, AJ, 140, 1868

Xilouris E. M., Madden S. C., Galliano F., Vigroux L., Sauvage M., 2004, A\&A, 416, 41

York D. G. et al., 2000, AJ, 120, 1579

Zubko V., Dwek E., Arendt R. G., 2004, ApJS, 152, 211

\section{APPENDIX A: THE MOCK CHECKS FOR THE EAGLE GALAXIES}

In this section, we perform the mock analysis which implies running additional CIGALE module that derives mock fluxes for each galaxy based on their respective best fit. Each flux is then varied in order to introduce noise, and these mock observations are then fitted again to derive the physical properties. In Fig. A1, results for nine properties are presented. The bottom row represents parameters associated with the THEMIS dust model: $\gamma$ is the fraction of the dust luminosity originating from PDRs, $q_{\mathrm{hac}}$ is the fraction of the total dust mass that is in small hydrocarbon grains, and $U_{\min }$ is the minimum intensity of the stellar radiation necessary the heat the dust grains.

The highest deviation is seen for $q_{\text {hac }}$, as found for the DustPedia sample (Nersesian et al. 2019). The rest of the parameters agree remarkably well, with the higher Spearman coefficients compared to the DustPedia sample (see fig. B.1 in Nersesian et al. 2019). The origin of the better correlation is the completeness of the flux data sets in the EAGLE-SKIRT data base (29 bands for all galaxies),
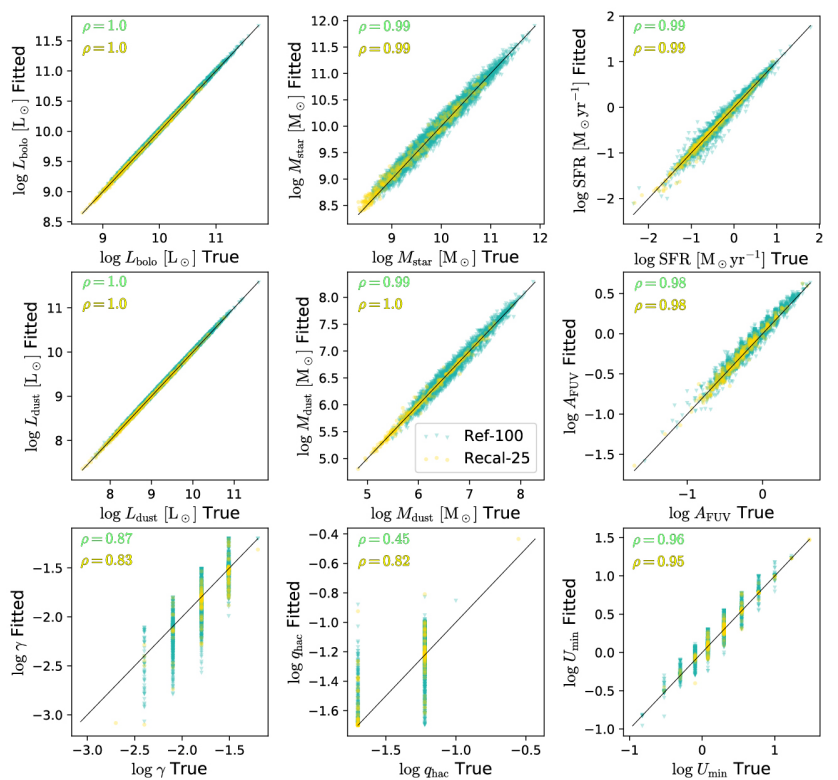

Figure A1. Results of the mock analysis by CIGALE for the two EAGLE samples. The Spearman coefficients are shown in the corners. The black line is one-to-one relation. 
contrarily to DustPedia where the median number of bands per galaxy is 20 (see Table 2).

${ }^{1}$ Sterrenkundig Observatorium, Universiteit Gent, Krijgslaan 281, B-9000 Gent, Belgium

${ }^{2}$ Leiden Observatory, Leiden University, PO Box 9513, NL-230 0 RA Leiden, the Netherlands

${ }^{3}$ INAF - Osservatorio Astrofisico di Arcetri, Largo E. Fermi 5, I-50125

Florence, Italy

${ }^{4}$ INAF - Istituto di Radioastronomia, Via P. Gobetti 101, I-40129 Bologna, Italy

${ }^{5}$ INAF - Istituto di Astrofisica Spaziale e Fisica Cosmica, Via Alfonso Corti 12, I-20133 Milan, Italy

${ }^{6}$ Department of Physics and Astronomy, University College London, Gower Street, London WC1E 6BT, UK

${ }^{7}$ School of Physics and Astronomy, Cardiff University, The Parade, Cardiff CF24 3AA, UK

${ }^{8}$ Instituto de Radioastronomía y Astrofísica, UNAM, Campus Morelia, A.P. 3-72, C.P. 58089, Michoacán, Mexico
${ }^{9}$ AIM, CEA, CNRS, Université Paris-Saclay, Université Paris Diderot, Sorbonne Paris Cité, F-91191 Gif-sur-Yvette, France

${ }^{10}$ Tsung-Dao Lee Institute, Shanghai Jiao Tong University, Shanghai 200240, China

${ }^{11}$ Department of Astronomy, Shanghai Key Laboratory for Particle Physics and Cosmology, Shanghai Jiao Tong University, Shanghai 200240, China

${ }^{12}$ Central Astronomical Observatory of RAS, Pulkovskoye Chaussee 65/1, 196140 St. Petersburg, Russia

${ }^{13}$ National Observatory of Athens, Institute for Astronomy, Astrophysics, Space Applications and Remote Sensing, Ioannou Metaxa and Vasileos Pavlou GR-15236 Athens, Greece

${ }^{14}$ Department of Astrophysics, Astronomy \& Mechanics, Faculty of Physics, University of Athens, Panepistimiopolis, GR-15784 Zografos, Athens, Greece

${ }^{15}$ Centre for Astrophysics Research, University of Hertfordshire, College Lane, Hatfield AL10 9AB, UK

This paper has been typeset from a $\mathrm{T}_{\mathrm{E}} \mathrm{X} / \mathrm{L} \mathrm{T}_{\mathrm{E}} \mathrm{X}$ file prepared by the author. 